\title{
Confronting Tipping Points: Can Multi-objective Evolutionary Algorithms Discover Pollution Control Tradeoffs Given Environmental Thresholds?
}

\author{
Victoria L. Ward ${ }^{\mathrm{a}}$, Riddhi Singh ${ }^{\mathrm{b}}$, Patrick M. Reed ${ }^{\mathrm{a}, *}$, Klaus Keller ${ }^{\mathrm{c}, \mathrm{d}, \mathrm{e}}$ \\ ${ }^{a}$ School of Civil and Environmental Engineering, Cornell University, Ithaca, NY, USA. Email: vlw27@cornell.edu, \\ patrick.reed@cornell.edu \\ ${ }^{b}$ Department of Civil Engineering, Indian Institute of Technology Hyderabad, Yeddumailaram, India. Email: \\ riddhi@iith.ac.in \\ ${ }^{c}$ Department of Geosciences, The Pennsylvania State University, University Park, PA, USA. Email: \\ klaus@psu.edu \\ ${ }^{d}$ Department of Engineering and Public Policy, Carnegie Mellon University, Pittsburgh, PA, USA. \\ ${ }^{e}$ Earth and Environmental Systems Institute, The Pennsylvania State University, University Park, PA, USA.
}

\begin{abstract}
This study contributes a stochastic, multi-objective adaptation of the classic environmental economics Lake Problem as a computationally simple but mathematically challenging benchmarking problem. The Lake Problem considers a hypothetical town by a lake, which hopes to maximize its economic benefit without crossing a nonlinear, and potentially irreversible, pollution threshold. Optimization objectives are maximize economic benefit, minimize phosphorus in the lake, maximize the probability of avoiding the pollution threshold, and minimize the probability of drastic phosphorus loading reductions in a given year. Uncertainty is introduced through a stochastic natural phosphorus inflow. We performed comprehensive diagnostics using six algorithms: the Borg multi-objective evolutionary algorithm (MOEA), MOEA/D, epsilon-MOEA, the Non-dominated Sorting Genetic Algorithm II (NSGAII), epsilon-NSGAII, and Generalized Differential Evolution 3 (GDE3) to evaluate their controllability, reliability, efficiency, and effectiveness. Our results show only the self-adaptive search of the Borg MOEA was capable of performing well on this nontrivial benchmarking problem.
\end{abstract}

Keywords: risk management, environmental thresholds, tipping points, multi-objective decision making, algorithm benchmarking, Lake Problem benchmark

${ }^{*}$ Corresponding author 


\section{Software and Data Availability}

- Description and Availability: The Lake Problem simulation code, code to replicate the diagnostics performed in this study, and best final reference sets are available on Github (https://github.com/VictoriaLynn/Lake-Problem-Diagnostics).The multi-objective evolutionary algorithm diagnostics can be replicated using the software code available for the MOEA framework (http://moeaframework.org/) and the Borg MOEA (http://borgmoea.org/).

- Developer: The simulation code was adapted by Victoria Ward from code developed by Riddhi Singh with contributions from Patrick Reed and Klaus Keller.

- Funding Source: Development of the code was partially supported by the National Science Foundation, through the Network for Sustainable Climate Risk Management (SCRiM) under NSF cooperative agreement GEO-1240507 as well as the Penn State Center for Climate Risk Management.

- Source Language: The simulation code is written in $\mathrm{C}++$.

- License: GNU Lesser General Public License, Version 3

\section{Introduction}

Over the last decade there has been an increased focus on the risks posed by high consequence thresholds or "tipping point" responses across a range of coupled environmental and economic systems (Kwadijk et al., 2010; Walker et al., 2013; Lenton, 2013; Kriegler et al., 2009; Brock, 2006; Keller et al., 2004). Tipping points represent a unique and often challenging class of environmental management problems because they often pose severe nonlinearities, high levels of uncertainty, irreversible consequences, and stakeholder tensions related to conflicting values or objectives. Haimes 
\& Hall (1977) provided one of the first explorations of these issues in the water resources planning and management literature. Their work strongly emphasizes the need for multi-objective analyses that are capable of capturing the sensitivity, stability, and irreversibility of candidate management actions. The core mathematical and decision support challenges that arise when managing environmental tipping points as eloquently framed by Haimes \& Hall (1977) remain as grand challenges today (for example, see the discussions in the following reviews: Herman et al. (In-Press); Walker et al. (2013); Nicklow et al. (2010); Reed et al. (2013); Keller et al. (2008)). The difficulties posed by environmental threshold problems have motivated their use as technical benchmarks when evaluating alternative decision support frameworks (Singh et al., 2015; Lempert \& Collins, 2007; Carpenter et al., 1999; Peterson et al., 2003; Kwadijk et al., 2010; Lenton, 2013; Brock, 2006; Hall et al., 2012). One of the most popular tipping point benchmark problems to emerge from the environmental decision-making literature is the Lake Problem (Carpenter et al., 1999; Peterson et al., 2003; Lempert \& Collins, 2007). It has a rich conceptualization that allows for a wide range of challenging management and mathematical traits to be explored with relatively modest computational demands.

The classical Lake Problem considers a fictional town on a lake seeking to balance competing desires of maximizing its economic productivity resulting in phosphorus pollution and minimizing the negative impacts on the lake's water quality. The current study builds on a specific instance of the Lake Problem where the lake's water quality is strongly impacted by a potentially uncertain threshold that governs transitions between two stable states: (1) an oligotrophic state (in simple terms, mostly unpolluted) or (2) a eutrophic polluted state (Carpenter et al., 1999). The transition from an oligotrophic to a eutrophic state can be rapid once the concentration of phosphorus in a lake reaches a critical threshold. Furthermore, the Lake Problem's simple but challenging system dynamics provide a high degree of flexibility in defining the consequences of crossing the water quality threshold.

In the arguably least challenging situation, the Lake Problem water quality impacts are reversible, meaning that the lake can be restored to an unpolluted state through reductions in phosphorus loading alone. In a more complex situation, the lake can show a hysteresis response, increasing the negative consequences of crossing the threshold, where restoration to an unpolluted state requires drastic reductions in pollution input. The most challenging case of the Lake Problem is defined such that the lake is irreversible, where water quality cannot be restored by a reduction 
in phosphorus loading alone. Consequently, if the town is situated on an irreversible lake there is an increased concern of crossing the threshold as the lake would then become permanently eutrophic. The Lake Problem's representation of decision making given the potential for crossing an irreversible threshold has made the problem a useful proxy for a broad class of environmental management problems (Brozović \& Schlenker, 2011; Carpenter et al., 1999; Lempert \& Collins, 2007). Simultaneously, it is useful for methodological benchmarking given its ability to represent tipping points, nonlinearity, and system uncertainties (Carpenter et al., 1999; Singh et al., 2015; Lempert \& Collins, 2007; Hadka et al., In-Press). Past analyses of the Lake Problem have typically approximated the decision making by the town as a single rational actor optimizing one weighted utility objective. This traditional normative economic formulation assumes an a priori preferential weighting between the town's economic benefits and the impacts of pollution (Carpenter et al., 1999; Lempert \& Collins, 2007; Peterson et al., 2003).

More recently, Singh et al. (2015) analyzed a multi-objective extension of the Lake Problem. In their extension, Singh et al. (2015) broaden the formulation to represent and analyze the tradeoffs among diverse stakeholder objectives (or values) representing near term versus long term economic benefits, an environmental regulatory perspective seeking to maximize water quality, and a reliability-based engineering perspective that seeks to minimize the probability of tipping the lake into an irreversible eutrophic state. This work illustrates how framing the problem as a maximization of expected utility (MEU) alone can lead to myopia and increase the risks of crossing the threshold response (Carpenter et al., 1999; Peterson et al., 2003; Lempert \& Collins, 2007).

Utility function formulations can prioritize economic benefits over other potential objectives, including environmental concerns. In systems confronting environmental thresholds, an a priori preferential weighting of economic benefits in utility functions may bias optimal policies to delay environmental management actions and increase the risks for an irreversible collapse in environmental quality (Admiraal et al., 2013; Peterson et al., 2003; Keller et al., 2004; McInerney et al., 2012). These prior studies explore the following concerns that emerge when using MEU to select environmental policies: (1) the MEU approach faces severe challenges in representing complex group or stakeholder preferences; (2) it is often difficult to understand the ecological and ethical ramifications of how MEU rankings value environmental systems; and (3) the MEU approach implicitly assumes that system dynamics and uncertainties are well characterized. Alternatively, Singh et al. (2015) illustrate the potential for multi-objective formulations to overcome the myopia of MEU formu- 
lations (Brill et al., 1990) by providing a far more diverse suite of management alternatives that explicitly compose the optimal trade-offs between economic and environmental objectives. This result reflects insights from a growing number of applications employing a posteriori multi-objective decision support, where decision makers explore key system tradeoffs before they have to express a preference for desired actions (Cohon \& Marks, 1975; Coello Coello, 2007; Nicklow et al., 2010; Reed et al., 2013; Maier et al., 2014).

A core requirement of the a posteriori multi-objective approach to managing environmental tipping points is the availability of solution techniques that are able to effectively approximate complex multi-objective tradeoffs (or Pareto fronts). Examples ranging from local management of landscape nutrient pollution (Brozović \& Schlenker, 2011; Carpenter et al., 1999; Lempert \& Collins, 2007; Peterson et al., 2003) to the impacts of crossing global climate change thresholds (Keller et al., 2004; Kriegler et al., 2009; Kwadijk et al., 2010), all demonstrate a strong tension or conflict between a range of economic, environmental, and inter-generational objectives or values. More formally, the solutions that define the Pareto optimal set (Cohon \& Marks, 1975; Pareto, 1896) for management applications can only be improved in one objective's performance by degrading their performance in one or more of the remaining objectives (i.e., the performance conflicts that yield tradeoffs). Plotting the Pareto optimal set of solutions in an application's objective space yields the Pareto front. Over the last 20 years, multi-objective evolutionary algorithms (MOEAs) have emerged as the dominant solution technique for supporting complex a posteriori multi-objective decision support (Coello Coello, 2007; Reed et al., 2013; Maier et al., 2014; Nicklow et al., 2010). Despite their rapidly growing popularity and broad application, Reed et al. (2013) have shown that stochastic many-objective (i.e., 3 to 10 objectives) risk-based planning applications with severe constraints can lead to search failures in a majority of currently available MOEAs.

This study builds off of the foundational MOEA benchmarking framework developed by Hadka \& Reed (2012b) as well as the specific insights for environmental and water resources provided by Reed et al. (2013) to demonstrate the value of our proposed many-objective version of the Lake Problem as a highly challenging benchmarking application. The contributed version of the Lake Problem is a high dimensional real-valued control problem (i.e., 100 decision variables) with significant performance constraints, stochastic uncertainty in natural phosphorus loading, and an irreversible environmental threshold. The problem combines the complexities of managing nonlinear tipping points and high-dimensional stochastic multi-objective control optimization to yield a 
benchmarking application that can pose severe challenges to modern solution techniques (see the reviews by Castelletti et al. (2008); Reed et al. (2013); Walker et al. (2013)). Building on the recent quantitative MOEA benchmarking and review by Reed et al. (2013), the Lake Problem benchmark problem and diagnostic assessment contributed in this study: (1) bridges a suite of severely challenging properties, (2) frames the current state-of-the-field using a statistically rigorous assessment, (3) demonstrates how even simple changes in how we represent systems' uncertainties can cause severe MOEA failures, and (4) can be extended beyond the benchmarking version presented in this study to test emerging frameworks for robust decision making (Hadka et al., In-Press; Singh et al., 2015). Overall, this study demonstrates that five of six tested modern MOEAs are not capable of supporting the management of uncertain environmental tipping points. The failures exhibited by these algorithms illustrate the importance of shifting towards recent advances in self-adaptive, multi-operator MOEA search frameworks (Hadka \& Reed, 2013, 2012a) in order for the environmental community to improve the multi-objective management of environmental tipping points.

\section{Lake Problem Formulation}

As briefly discussed in Section 1, our proposed benchmarking variant of the Lake Problem abstracts a town near a lake that must determine an annual phosphorus pollution control strategy that seeks to maximize its economic benefits from pollution without triggering irreversible eutrophication

(Figure 1). The classic formulation of this problem was developed by Carpenter et al. (1999) and uses a dimensionless differential equation shown in equation (1) describing the dynamic evolution of phosphorus in the shallow lake over time.

$$
\begin{array}{r}
P_{t}=P_{t-1}+a_{t}-b P_{t-1}+\frac{P_{t-1}^{q}}{1+P_{t-1}^{q}}+\epsilon_{t} \\
\epsilon_{t} \sim \operatorname{lognormal}(\mu, \sigma)
\end{array}
$$




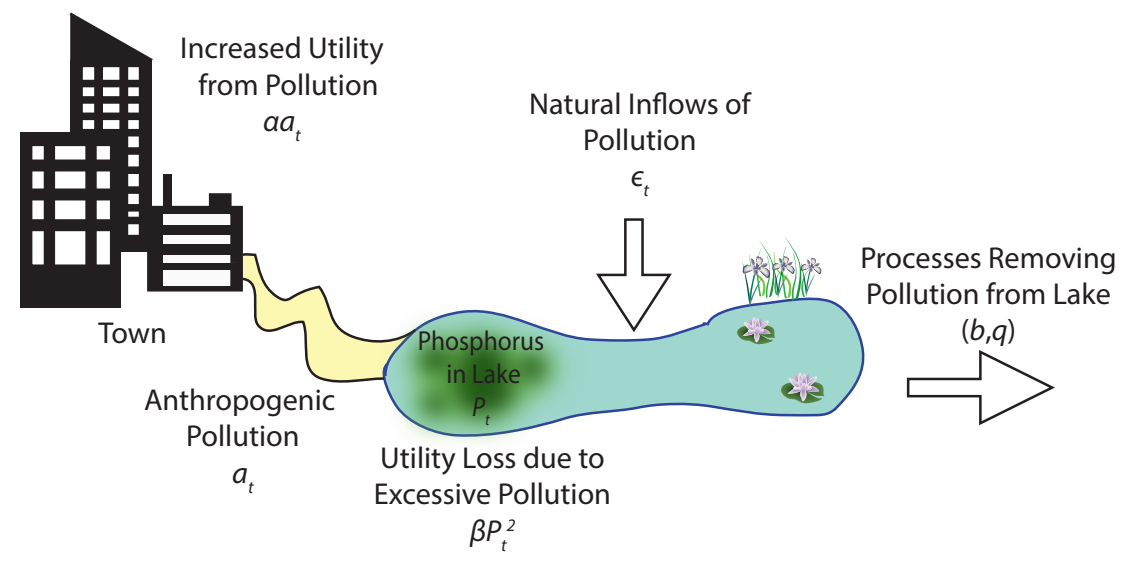

Figure 1: Illustrative overview of the Lake Problem showing possible inflows and outflows of phosphorus to the lake (adapted from Hadka et al. (In-Press)). Anthropogenic phosphorus pollution enters the lake as a byproduct of the town's industrial activities, adding to stochastic natural inflows of pollution from the environment. The lake is able to remove part of this pollution based on its properties, but excessive accumulation of phosphorus leads to algal blooms that decrease the utility of the lake (reduced tourism, fishing, etc.).

In equation (1), $P_{t}$ represents the mass concentration of phosphorus (dimensionless) in the lake at annual time step $t, P_{t-1}$ is the mass concentration of phosphorus in the lake at the previous time step (dimensionless), $a_{t}$ is the town's phosphorus loading (dimensionless) at time step $t$, and $b$ and $q$ are parameters of the lake model that determine whether eutrophic states in the lake are irreversible, reversible, or hysteretic. Our chosen parameter values, including the phosphorus recycling rate, $q$, and phosphorus decay rate, $b$, are provided in Table 1 . We use values for $q$ and $b$ that represent an irreversible lake, which will remain in a polluted (or eutrophic) state permanently once the lake tips past a critical pollution threshold (Carpenter et al., 1999). The last term represents uncertain natural phosphorus inflows to the lake, which are here represented through a lognormal distribution. This term was motivated by the original formulation by Carpenter et al. (1999) to introduce uncertainty in an uncontrolled phosphorus loading (e.g., nonpoint source pollution). 
Table 1: Parameters for the lake model simulation and optimization

\begin{tabular}{|c|c|c|c|c|}
\hline Category & Name & Parameter & Value & Dimensions \\
\hline \multirow[t]{4}{*}{ Lake Model } & Phosphorus Removal Rate & $\mathrm{b}$ & 0.42 & dimensionless \\
\hline & Steepness of Sigmoid Curve & $q$ & 2 & dimensionless \\
\hline & Number of Years & $\mathrm{T}$ & 100 & years \\
\hline & Number of Samples & $\mathrm{N}$ & 100 & dimensionless \\
\hline \multirow{3}{*}{$\begin{array}{l}\text { Benefit } \\
\text { Estimation }\end{array}$} & Cost Multiplier & $\alpha$ & 0.4 & dimensionless \\
\hline & & & & \\
\hline & Discount Factor & $\delta$ & 0.98 & dimensionless \\
\hline Uncertainty & Total number of stochastic samples & & 10000 & dimensionless \\
\hline Estimation & for each distribution & & & \\
\hline $\begin{array}{l}\text { Reliability } \\
\text { Estimation }\end{array}$ & Critical Phosphorus Level & $X_{c r i t}$ & 0.5 & dimensionless \\
\hline $\begin{array}{l}\text { Inertia } \\
\text { Estimation }\end{array}$ & Critical reduction in phosphorus & $I_{\text {crit }}$ & -0.02 & dimensionless \\
\hline Optimization & Phosphorus in lake & $\varepsilon_{\text {phosphorus }}$ & 0.01 & dimensionless \\
\hline$\varepsilon$-values & & & & \\
\hline & Economic Benefit of Pollution & $\varepsilon_{\text {benefit }}$ & 0.01 & dimensionless \\
\hline & Reliability & $\varepsilon_{\text {reliability }}$ & 0.0001 & dimensionless \\
\hline & Inertia & $\varepsilon_{\text {inertia }}$ & 0.0001 & dimensionless \\
\hline
\end{tabular}

Our formulation considers environmental management tradeoffs for two natural phosphorus inflow scenarios. The first, Scenario 1, involves a lognormal distribution with a real-space (as opposed to log-space) mean of 0.02 and a $\log 10$ variance of -5.5 . The second, Scenario 2 , is more challenging with a real-space mean of 0.03 and a $\log 10$ variance of -5 . These scenarios represent the extremes of low mean-low variance (well-informed uncertainty) and high mean-high variance (poorly informed uncertainty) scenarios, enabling us to assess the impact of changing the uncertainty assumption on 
our results. Our selection of the lognormal distributions that define Scenarios 1 and 2 builds on our prior detailed analyses in Singh et al. (2015) where three alternative problem formulations and nine alternative lognormal distributions for natural phosphorus inflows were analyzed for their relative impacts on the identification of pollution control strategies as well as the potential for crossing the lake's tipping point. For the purposes of this study, moving from Scenario 1 to Scenario 2 illustrates the algorithmic impacts of increasing the likelihood of irreversibly crossing the lake's tipping point. We begin the simulations with an unpolluted lake by assuming an initial condition of $P_{0}=0$. Equation (1) is simple enough to be easily understood while also capturing the threshold behavior of lake eutrophication.

Carpenter et al. (1999) represents the town's preference for balancing economic benefits associated with phosphorus pollution with the desire to maintain the lake's ecosystem services by limiting pollution. They consolidate these goals in a single objective optimization where the town seeks to maximize the expected utility function shown in equation (2) below. The first term in equation (2) is a positive, linear term representing the economic benefit to the town associated with phosphorus pollution. The second term is a negative quadratic penalty term that accounts for the negative impact of losing ecosystem services on the net economic benefit associated with phosphorus loading.

$$
U t i l_{t}=\alpha a_{t}-\beta P_{t}^{2}
$$

In equation (2), Util $l_{t}$ is the undiscounted value of the town's utility at a given time step (dimensionless), $\alpha$ is the willingness of the town to pay for the right to export an additional unit increment of phosphorus pollution (dimensionless), $\beta$ is the compensation lake users are willing to accept to tolerate a given state (dimensionless), $a_{t}$ is the anthropogenic phosphorus load at time step (dimensionless), $t$, and $P_{t}$ is the amount of phosphorus in the lake at time step (dimensionless), $t$. In Carpenter et al. (1999), $\alpha$ is fixed at 0.4 and $\beta$ at 0.08 representing an a priori weighting (Clemen \& Reilly, 2001; Coello Coello, 2007; Cohon \& Marks, 1975; Köksalan et al., 2011) of the relative conflicting objectives of maximizing economic benefits by polluting the lake versus minimizing the phosphorus concentration in the lake.

In this study, we modify the Lake Problem formulation to be a four objective problem. In the new formulation, we seek to explore the tradeoffs that emerge when maximizing the economic benefits from pollution, minimizing phosphorus concentrations in the lake, maximizing the reliability of pollution control strategies in avoiding the threshold above which the lake enters an irreversible 
eutrophic state, and maximizing the probability of avoiding extreme changes in pollution control strategies from year to year (e.g., avoid rapidly shutting down all of the town's economic activity or making massive investments in abatement). Moving to a multi-objective formulation provides more contextual support for understanding the town's tradeoffs (Singh et al., 2015) while also clarifying the environmental consequences of a sole focus on maximizing expected utility (Admiraal et al., 2013; Peterson et al., 2003). Equations (3)-(5) provide a more formal symbolic representation of our proposed Lake Problem formulation, which optimizes a vector of four objectives, $F(a)$, using the control vector of environmental control decision variables, a. Note detailed definitions and descriptions of the four objectives in equation (3) are provided later in this section.

$$
\begin{gathered}
F(a)=\left(O_{1}, O_{2}, O_{3}, O_{4}\right), \\
a=\left(a_{1}, a_{2}, \ldots, a_{100}\right) \\
\text { subject to, } c_{r e l}: O_{4}>0.85
\end{gathered}
$$

In equation (4), $a_{t} \in[0,0.1]$ represents the amount of phosphorus the town decides to emit in each time step, $t$, from year 1 to 100 years. The reliability constraint, $c_{r e l}$, requires that the town avoid tipping the lake into an irreversible eutrophic state $85 \%$ of the time. This reliability is calculated based on the state of the lake across all uncertain States of the Worlds (SOWs) and time periods. It is worth noting that although the instance of the Lake Problem introduced here represents a highly challenging benchmark as will be shown in our results, the reliability objective assumes knowledge of the lake's tipping point concentration, and the $85 \%$ reliability constraint reflects more risk tolerance than has typically been accepted in the recent water management literature (Characklis et al., 2006; Kasprzyk et al., 2009; Zeff et al., 2014). Additionally, we assume perfect knowledge of the lake parameters, phosphorus concentration in each time step, and the town's economic objectives. In real planning contexts, defining the planning objectives, decision actions, constraints and key system uncertainties represents a critically important challenge as has been long recognized (Brill et al., 1990; Hitch, 1960; Kasprzyk et al., 2012; Liebman, 1976; Reed \& Kasprzyk, 2009; Tsoukias, 2008).

During the optimization, each candidate pollution control strategy is evaluated using a Monte 
Carlo lake simulation for 100 SOWs drawn randomly from the lognormal distributions of the natural phosphorus inflows. The choice of using 100 SOWs for each Monte Carlo simulation-based function evaluation represents a balance between computational demands and the ability of the MOEAs to search effectively given noisy evaluations of objectives. Each Monte Carlo function evaluation as implemented in this study is based on a random draw of 100 out of 10,000 SOWs (see Table 1) for natural phosphorous inflows. Assuming the MOEA is functional under noisy evaluations, our analysis has shown evolutionary search with 100 SOWs can yield Lake Problem management strategies whose performance remain largely unchanged even when re-evaluated later with substantially larger draws (e.g., the 10,000 SOWs) from the lognormal distributions of the natural phosphorus inflows. Our use of noisy evolution based on 100 SOWs is very similar to a large body of work related to evolutionary optimization under uncertainty (Chan Hilton \& Culver, 2005; Fu \& Kapelan, 2011; Gopalakrishnan et al., 2001; Kasprzyk et al., 2009; Singh et al., 2010; Smalley et al., 2000; Wu \& Whittington, 2006; Zeff et al., 2014). The Lake Problem tests the capability of MOEAs to explore an extremely high-dimensional, severely nonlinear, and stochastic environmental control problem. Reed et al. (2013) showed that many modern algorithms can struggle with stochastic, many-objective applications. The Lake Problem, as formulated in this study, distinguishes itself from prior MOEA benchmarking problems given its high-dimensional pollution control decisions and the potential for irreversible tipping points.

Expected Economic Benefits: The town's economic benefits objective $O_{1}$ is computed as the expected positive economic benefit associated with phosphorus loading summed over all time steps. We calculate this objective using the first term in the utility function found in Carpenter et al. (1999).

$$
\text { Maximize } O_{1}=\sum_{t=1}^{T} \alpha a_{t} \delta^{t}
$$

The economic benefits in equation (6) are associated with phosphorus loading from the town. Note that equation (6) abstracts the economic value the town places on being permitted to discharge phosphorus and represents a stochastic time discounted generalization of the first term of the original utility function used by Carpenter et al. (1999) shown in equation (2) above. The expected value of $O_{1}$ is computed over $T$ time steps, where $t$ is the index for the current time step and $\alpha$ is an economic parameter fixed at 0.4 , capturing the willingness of the town to pay for pollution. 
The pollution decision variable, $a_{t}$ is the amount of phosphorus loading at time step $t$, and $\delta$ is the discount factor used to translate future to present utilities, which was fixed at 0.98 in this formulation (Carpenter et al., 1999; Singh et al., 2015).

Worst Case Average Phosphorus Concentration: In the expected utility maximization formulation used originally by Carpenter et al. (1999) and summarized in equation (2) above, they used a weighted quadratic penalty term to abstract water quality as an objective. Alternatively, we capture environmental values in equation (7) below by seeking to minimize the maximum average phosphorus concentrations across all time steps. Minimization of the maximum average across time steps was used to capture the town's environmental goals because it accounts for the accumulation of phosphorus over longer time periods. Further, it is a conservative calculation but less conservative than a minimization of the maximum phosphorus concentration in any state of the world and time step, which may place undue emphasis on high natural phosphorus inflows with a very low probability of occurring. In direct conflict with the economic benefits objective in equation (6), this objective seeks to minimize the worst case average accumulation of phosphorus in the lake.

$$
\begin{array}{r}
\text { Minimize } O_{2}=\max P_{t} \text { for } 1 \leq t \leq T \\
P_{t}=\frac{1}{N} \sum_{i=1}^{N} p_{i, t}
\end{array}
$$

As shown in equation (7), the average phosphorus in the lake at each time step is averaged over $N$ SOWs. The index, $i$ refers to each of the sampled SOWs drawn from the lognormally distributed uncertain phosphorus inputs. The total contributed phosphorus in the lake at time $t$ in SOW $i$ is represented as $p_{i, t}$.

Limits on Maximum Inter-Annual Pollution Reductions: Reflecting the fact that the town does not want extremely rapid reductions in phosphorus pollution through rapid investment in phosphorus removal or reductions in phosphorus-producing economic activity, equation (8) seeks to minimize the fraction of planning years where inter-annual pollution reductions $\left(O_{3}\right)$ between any two periods are more than $20 \%$ of the maximum possible reduction. Satisfying this maximum inter-annual reduction requires a minimum of five years to institute the maximum feasible pollution control, restricting the rate of growth of abatement to reflect political and infrastructural inertia. Although the inertia objective encourages that the town's annual reductions of phosphorous pollution be $20 \%$ or less, this limit is not strictly enforced as a deterministic maximum control rate. 
Treating it as a probability of compliance, allows the town to retain the capability of rapid pollution reductions while yielding a more diverse suite of potential control strategies. By allowing aggressive control strategies to remain feasible, we refrain from making an a priori assumption regarding decision maker preferences. This objective adds an inter-annual time scale (or short term) concern to the long-term management objectives while abstracting limits in response common in environmental management.

$$
\text { Maximize } O_{3}=\frac{1}{T} \sum_{t=1}^{T} \phi_{t} \text { where } \phi_{t}=\left\{\begin{array}{l}
1 \text { if } a_{t}-a_{t-1}>I_{\text {limit }} \\
0 \text { if } a_{t}-a_{t-1} \leq I_{\text {limit }}
\end{array}\right.
$$

In equation (8), $\phi_{t}$ is the inertia index at a given time step, $t$, which is 1 if the reduction in phosphorus loading between adjacent time steps is less than the specified limit $\left(I_{\text {limit }}\right)$ and 0 otherwise. The reduction limit is set at -0.02 , so the difference between loading in the current and previous time steps must be greater than this value for the reduction to be less than $20 \%$ of the maximum possible range, which was 0 to 0.1 .

Reliability of Avoiding Lake's Tipping Point: Equation (9) seeks to maximize the probability that the phosphorus concentration in the lake remains below the critical threshold reflecting the town's desire to avoid eutrophication of the lake. The value of this objective is penalized for every time step in every SOW for which the concentration of phosphorus in the lake is higher than the threshold.

$$
\text { Maximize } O_{4}=\frac{1}{N T} \sum_{i=1}^{N} \sum_{t=1}^{T} \theta_{t, i} \text { where } \theta_{t, i}=\left\{\begin{array}{l}
1 \text { if } P_{t, i}<P_{c r i t} \\
0 \text { if } P_{t, i} \geq P_{c r i t}
\end{array}\right.
$$

In equation (9), $\theta_{t, i}$ is the reliability index at a given time step, $t$, and state of the world, $i$, which is 1 if the phosphorus in the lake is below the specified critical threshold $\left(P_{\text {crit }}\right)$ and 0 otherwise. The critical threshold approximates the minimum pollution value at which the lake transitions from an unpolluted to polluted stable state and is set at 0.5 based on the parameters of the lake model (Singh et al., 2015). Reliability is more generous here than it would be in a real world scenario as there is a constraint that reliability must remain above $85 \%$, implying that the lake can be flipped to a eutrophic state in $15 \%$ of the cases. In real decision contexts, decision makers often seek to achieve $100 \%$ reliability (Caldwell \& Characklis, 2014), which would increase the difficulty of this already challenging problem. Furthermore, in this formulation, the town knows the value of the 
threshold at which the lake will flip. In reality, there is uncertainty in this value as explored in Hadka et al. (In-Press).

\section{Modern Multi-Objective Evolutionary Algorithm Suite}

\subsection{Algorithm Selection}

The use of MOEAs in environmental and water resources management is becoming common across a myriad of application areas given the common occurrence of large numbers of conflicting objectives as well as other mathematical complexities such as nonlinearity, stochasticity, discreteness, non-convexity, high dimension decisions, severe combinatorial growth rates and uncertainty (Maier et al., 2014; Nicklow et al., 2010; Reed et al., 2013). The growing popularity of MOEAs in water resources can be strongly attributed to the Non-dominated Sorting Genetic Algorithm-II (NSGAII) (Deb et al., 2002b; Reed et al., 2003), which has been widely applied due to its elitism, efficient non-dominated sorting, and parameter free diversity maintenance, which improved the range of problems where diversity and convergence could be sustained. Since NSGAII, a new generation of MOEAs has emerged and dramatically increased the size and scope of multiobjective problems that can be addressed. In spite of this progress, many modern algorithms have not been rigorously benchmarked on environmental problems that encompass the complexities inherent to our formulation of the Lake Problem. To address this concern, this study contributes a careful assessment of the effectiveness, efficiency, reliability, and controllability of six benchmark MOEAs on the four objective formulation of the Lake Problem. This diagnostic assessment builds on that of Reed et al. (2013) by performing algorithm diagnostics on our proposed many-objective version of the Lake Problem, representing a new problem class encompassing high-dimensional stochastic control in the presence of irreversible environmental tipping points. The six algorithms used in this study were the Borg MOEA, MOEA/D, GDE3, NSGAII, $\varepsilon$-NSGAII, and $\varepsilon$-MOEA (Table 2).

The reader may note that the six algorithms selected in this diagnostic study are a subset of those tested in the prior benchmarking studies by Hadka \& Reed (2012a) and Reed et al. (2013). Two factors informed our choice for inclusion in this study: (1) algorithmic computational cost and (2) prior demonstrated success on published benchmarks. The excluded MOEAs (e.g., the Strength Pareto Evolutionary Algorithm II or the Hypervolume Indicator Based Evolutionary Algorithm) have algorithmic components that are severely inefficient and that would increase the wall clock 
time of globally sampling their parameter spaces by 2-3 orders of magnitude (i.e., weeks of serial computing become months to years). Moreover, they have not established themselves consistently as top performers, especially for prior nonlinear, stochastic problems (Reed et al., 2013).

Table 2: Enumeration of algorithms used in the study

\begin{tabular}{lll}
\hline Algorithm & Class & Reference \\
\hline Borg MOEA & Adaptive multi-operator & Hadka and Reed (2013) \\
$\varepsilon-M O E A$ & Pareto front approximation & Deb et al. (2005) \\
$\varepsilon-N S G A I I$ & Pareto front approximation & Kollat and Reed (2006) \\
MOEA $/ D$ & Aggregate functions & Zhang et al. (1999) \\
GDE3 & Differential evolution & Kukkonen and Lampinen (2005) \\
NSGAII & Baseline & Deb et al. (2002) \\
\hline
\end{tabular}

\subsection{Algorithm Suite used in this Study}

NSGAII (Deb et al., 2002b) was chosen as it is the most popular multi-objective evolutionary algorithm in the world (Reed et al., 2013) and can be viewed as an important historical baseline algorithm against which the other five, more recent, algorithms may be compared. It uses the Pareto dominance relation to search for the entire Pareto front in a single run. NSGAII provided three key innovations: (1) a fast nondominated sorting procedure, (2) a fast crowded distance estimation procedure, and (3) a simple crowded comparison operator. Prior to NSGAII, the use of multi-objective evolutionary algorithms had been inhibited by high computational complexity, a lack of elitism, and the need to specify a sharing parameter. As a brief example of elitism, NSGAII selects the best $M$ solutions from a combined pool of $M$ parents and $M$ children. The children must outperform the parent solutions to be retained from the $2 M$ combined set of solutions. This selection of the top $M$ from $2 M$ solutions makes NSGAII sensitive to its population size. By introducing elitism, NSGAII expedited the search process and reduced the loss of good solutions found in prior generations (although this remains a limitation). In order to make the algorithm more userfriendly and computationally simple, NSGAII replaced the user-specified sharing parameter with a crowd-comparison approach, which employs a crowded-comparison operator to maintain diversity in the Pareto-optimal front. This operator gives preference to nondominated solutions located in less crowded regions to ensure the Pareto-optimal front is spread out. Another innovation accompanying 
the development of NSGAII was constraint-handling, which increased the pragmatism of applying MOEAs to real-world problems that are often constrained (Deb et al., 2002b).

$\varepsilon$-NSGAII (Kollat \& Reed, 2006) is based upon the original NSGAII (Deb et al., 2002b) but incorporates epsilon dominance archiving (Laumanns et al., 2002) and adaptive population sizing to improve search efficiency and reliability as well as the algorithm's ease of use for water resources applications. The addition of these features has been shown to reduce some of the need for extensive parameter calibration (Hadka \& Reed, 2012a; Kollat \& Reed, 2006; Tang et al., 2006). Epsilon dominance features a user-specified $\varepsilon$ grid applied to the search space of the problem. The value of $\varepsilon$ is based on the desired precision of the objective values for their particular application. If more than one solution falls in a given grid block, the solution closest to the lower left-hand corner of the block is chosen (assuming minimization of all objectives). Non-dominated sorting occurs based on the grid blocks, resulting in a thinning of solutions that increases diversity by promoting a more even search of the objective space without adding any additional parameters to the algorithm. The $\varepsilon$-NSGAII initially employs a small population size to direct search at a low computational cost until the algorithm begins to find fit solutions. Once highly fit solutions have been found, selection pressure is increased substantially by increasing the population size. Another feature of $\varepsilon$-NSGAII is the injection of epsilon non-dominated solutions from the archive to make up $25 \%$ of the subsequent population while the other $75 \%$ of the population is generated randomly. In the limit when the epsilon dominance archive size stabilizes, the series of connected runs resulting from the adaptive population sizing described above are equivalent to a diversity-based EA search enhancement termed time continuation (Goldberg, 2002).

$\varepsilon$-MOEA (Deb et al., 2005) is another example of an epsilon dominance algorithm, which increased the pragmatism of MOEAs by attaining a good approximation of the Pareto front quickly. It was the first MOEA to use $\varepsilon$ dominance to maintain an approximation of the Pareto front through an archive that adaptively grows and shrinks. The $\varepsilon$-MOEA was a breakthrough algorithm in that it reduced the tradeoff between efficiency and the quality of the Pareto approximate front by using efficient parent and archive update strategies to attain a well-distributed and well-converged set of solutions quickly. It evolves two populations, an archive and a parent, simultaneously and independently. Using one solution from each population, offspring solutions are generated using the simulated binary crossover (SBX) recombination operator (Deb \& Agrawal, 1994) and the polynomial mutation (PM) operator (Deb \& Goyal, 1996) to update the archive population based 
on $\varepsilon$-dominance. Traditional dominance is used to update the parent population. The $\varepsilon$-MOEA is a steady state algorithm in that an offspring competes with only one parent to take its slot in the search population in each evolution cycle through mating, mutation, and selection.

MOEA/D (Zhang et al., 2009) was chosen as a representative of the emerging class of decomposition MOEAs (Giagkiozis \& Fleming, 2014), which exploit populations of weighted single-objective transformations of multi-objective problems. Each solution of the multi-objective Pareto front approximated by an aggregate function is an optimal solution of a single objective function in which the objective is a linear or nonlinear aggregation function of all the individual objectives. MOEA/D accomplishes this aggregation of many-objective problems into a single-objective problem by simultaneously solving many single-objective Chebyshev decompositions of the many-objective problems. MOEA/D employs dynamical resource allocation by assigning different amounts of computational time to different sub-problems. MOEA/D defines neighborhood relations among the single objective sub-problems based on the distances among their weight vectors and optimizes each sub-problem based mainly on information from neighboring sub-problems. MOEA/D takes the problem, number of sub-problems to consider, a uniform spread of $\mathrm{N}$ weight vectors, and number of vectors in the neighborhood of each weight vector as inputs. This algorithm has established itself as a benchmark by winning the 2009 IEEE Congress on Evolutionary Computation (CEC 2009) competition (Zhang \& Suganthan, 2009).

GDE3 (Kukkonen \& Lampinen, 2009) is a multiobjective variant of the differential evolution (DE) algorithm for global optimization with an arbitrary number of objectives and constraints. The DE algorithm was introduced by Storn \& Price (1997) based on a desire for simplicity, efficiency, and the use of floating point encoding instead of binary numbers. In each generation, DE goes through each decision vector of the population to create a corresponding trial vector. The scaled difference between two randomly chosen vectors defines magnitude and direction of the mutation. When the difference is added to a third randomly chosen vector, this corresponds to a mutation of the third vector. Users define parameters controlling the crossover operation and the scaling factor for mutation. The crossover parameter controls the rotational invariance of the search with larger values being beneficial to non-separable problems. The value of the mutation scaling factor controls the speed and robustness of the search, requiring one to strike a balance between efficiency and premature convergence. Selection at the end of each generation is based on comparison of the trial vector to the old vector. If both vectors are feasible and neither dominates the other, both 
vectors are chosen for the next generation, meaning the size of the population can increase after a generation. To trim the population, a selection approach similar to that of NSGAII is employed where vectors are sorted based on non-dominance and crowdedness. A benefit of the operator employed by this algorithm is that it is rotationally invariant, meaning it does not assume explicit search directions when it creates new solutions. This allows for decisions that have conditional dependence.

Borg MOEA (Hadka \& Reed, 2013) is not a single algorithm, but a self-adaptive evolutionary search framework whose search operators are selected based on their effectiveness in a given problem's search domain. The Borg MOEA builds on the $\varepsilon$-MOEA algorithm introduced by (Deb et al., 2005) as its parent algorithm. The use of $\varepsilon$-MOEA's steady state algorithm architecture gives the Borg MOEA a highly efficient design that can be exploited for massive parallelization (Reed \& Hadka, 2014; Hadka \& Reed, In-Press). In addition to the steady-state elitist model of $\varepsilon$-MOEA, the Borg MOEA features $\varepsilon$-dominance, $\varepsilon$-progress, an adaptive population sizing operator similar to $\varepsilon$-NSGAII's (Kollat \& Reed, 2006) use of time continuation to maintain search diversity and to facilitate escape from local optima, and multiple recombination operators. The Borg MOEA measures $\varepsilon$-progress based on whether recently discovered solutions have dominated one or more solutions in the epsilon dominance archive. If the algorithm fails to make sufficient $\varepsilon$-progress in one generation, it will automatically trigger a restart operator to avoid search stagnation. When a restart is triggered, the search population is adapted to remain proportional to the epsilon-dominance archive size; the tournament size is adapted to maintain consistent selection pressure, and uniform mutation is used to diversify the search population with a 4:1 ratio of new mutated members to archive solutions. The recombination operators are rewarded based on their generation of new archive members in that their probability of use is based on the number of solutions produced by each operator in the $\varepsilon$-box dominance archive and updates throughout the algorithm's search. The primary search operators used by the Borg MOEA are simulated binary crossover (SBX; (Deb \& Agrawal, 1994)), differential evolution differencing (DE; (Storn \& Price, 1997)), parent-centric crossover (PCX; (Deb et al., 2002a)), unimodal normal distribution crossover (UNDX; (Kita et al., 1999)), simplex crossover (SPX; (Tsutsui et al., 1999)), and uniform mutation (UM). Offspring produced by SBX, DE, PCX, UNDX, and SPX are mutated using polynomial mutation (PM; (Deb \& Agrawal, 1994)). The Borg MOEA's endogenous auto-adaptive search processes have been designed to enhance its applicability across a wide set of problem classes while 
reducing its sensitivity to its underlying parameterization (Hadka \& Reed, 2012a; Hadka et al., 2012; Reed et al., 2013).

\section{Diagnostic framework}

\subsection{Computational experiment}

Figure 2 adapted from Reed et al. (2013) provides an illustrative schematic of the diagnostic assessment framework used in this study to statistically compare the performance of the six algorithms used to benchmark this four-objective formulation of the Lake Problem. The figure illustrates the diagnostic protocol we implemented for each algorithm. Because MOEAs are stochastic search tools that range in their use and parameterization of operators (i.e., mating, mutation, selection, archiving, etc), this diagnostic assessment avoids making specific assumptions regarding optimal or highly tuned parameters for each algorithm by sampling the full feasible parameter space for each algorithm using Latin Hypercube Sampling (LHS) as depicted in the parameter blocks shown in Figure 2. Each of the points depicted in the parameter blocks represents a full specification of a candidate MOEA's parameters that are run to attain its corresponding approximation set for a given problem. 


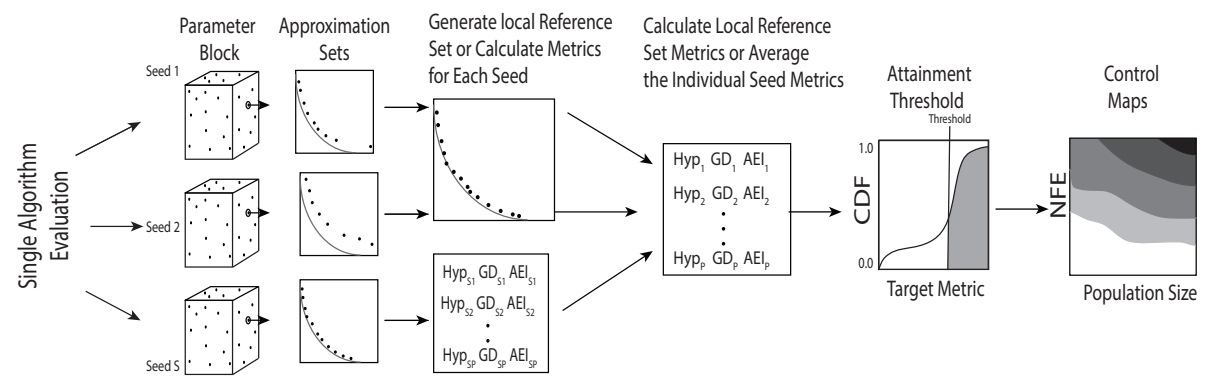

Figure 2: Diagnostic assessment framework used to evaluate each of the six tested MOEAs (adapted from Reed et al (2013) and Hadka and Reed (2012)). For each algorithm, a Latin Hypercube sample of its parameters across their full feasible ranges is used to generate the parameter block. Each parameter set in the parameter block is evaluated by running an MOEA to attain the resulting approximation sets. Random seed effects are managed by using S replicate trials to ensure the statistical quality of our results. From the resulting approximation sets, this study computes two alternative versions of performance metrics. The first determines average metric values attained for each parameterization of each algorithm, representing the expected performance if an MOEA is run for a single random seed trial. The second generates local reference sets for each parameterization across its random seed trials before calculating metrics. The resulting local reference sets represent MOEA performance given the standard use of random seed analysis. Given the global sampling of potential MOEA performance, attainment thresholds can be used to explore cumulative distribution functions (CDFs) for different measures of performance. Control maps of performance are shown as projections of number of function evaluations (NFE) and population size to capture the efficiency and controllability of each algorithm.

This study used LHS to test 500 parameterizations drawn across the full feasible ranges of each MOEA's potential parameterization space. Each parameter draw was used to optimize the four objective variant of the Lake Problem under both natural inflow scenarios. To further account for the stochastic nature of MOEAs, the runs of the LHS parameter block were replicated for 50 random number generator seeds to account for the fact that random effects in initial populations and operators can impact algorithm performance. For each approximation set, several computed metrics measure its convergence, consistency, and diversity (See section 4.2). For the proposed Lake Problem benchmarking problem, the true global Pareto fronts are unknown. Therefore, best 
known approximations were generated from all runs of all six algorithms for each instance of the problem and used as reference sets in our metric calculations. In this study, the two instances of the problem were identical with the exception of the natural inflow distribution, which was enough to alter the problem difficulty substantially. Consequently, there were two separate reference sets corresponding to the two different distributions of natural phosphorus inflows.

Each approximate front was compared to the appropriate reference set in the calculation of metrics. Two approaches were taken in calculating these metrics as depicted in Figure 2. The first approach accounted for random seed effects through the generation of a local reference set for each parameterization of each algorithm. This represents the standard case of running an MOEA for multiple random seed trials and developing a local reference set. These local reference sets were generated by performing an epsilon non-dominance sort across the solutions found by all random seeds for each parameterization of every algorithm. Note that to be consistent when comparing point dominance algorithms such as NSGAII with algorithms that exploit epsilon-dominance that reference sets and metrics need to be computed using a consistent epsilon non-dominance sorting. It is possible to convert point dominance into epsilon-dominance, but the reverse is never true which can lead to biased metrics (Hadka \& Reed, 2013, 2012a; Reed et al., 2013). All subsequent MOEA diagnostic metrics were calculated by comparing each local reference set to the global reference set. The second approach for assessing MOEA performance characterized the expected performance from running an MOEA once using a single random seed. In this case, metrics were calculated by comparing every approximation set to the global reference set. These metrics were then averaged across all seeds for each parameterization of each algorithm.

As Figure 2 shows, attainment thresholds (i.e., goal metric values) were used to quantify the probabilities of successful search across all runs and provide insight to the effectiveness and reliability of the MOEAs used in this study on the Lake Problem. Effectiveness reflects the ability of an algorithm to attain a good approximation to the Pareto front while reliability indicates how consistently an algorithm achieves a high level of performance. As Figure 2 illustrates, we also used control maps developed from our LHS of parameterizations to assess the controllability and efficiency of the algorithms studied by showing a sub-space projection plot of performance for population size vs. the number of function evaluations (NFE) used in search. Overall, population size has a dominant impact on MOEA performance, and NFE provides insight into algorithm efficiency or how quickly an algorithm attains a decent approximation to the Pareto front. Controllability 
reflects the fact that parameter selection can have significant impacts on the success or failure of an algorithm. When an algorithm is well suited to a problem, almost any parameter combination will yield an acceptable performance, which is indicated by a large swath of high performance on its control map. Goldberg (2002) referred to this as a large "sweet spot" in the parameter space of an algorithm. Conversely, when an algorithm is not well suited to a problem or that problem is particularly difficult, selecting a successful parameterization may be extremely difficult, indicating full algorithmic failure. Table 3 provides a detailed summary of the operators, parameter ranges, and LHS sampling used in this study. 
Table 3: Latin Hypercube Sampling of algorithm operators and their associated parameter ranges.

\begin{tabular}{|c|c|c|c|}
\hline & Parameter & Range & Algorithm(s) \\
\hline \multirow[t]{15}{*}{ Crossover } & SBX Rate & $0.0-1.0$ & Borg, $\varepsilon$-MOEA, $\varepsilon$-NSGAII, NSGAII \\
\hline & SBX Distribution Index & $0.0-500.0$ & Borg, $\varepsilon$-MOEA, $\varepsilon$-NSGAII, NSGAII \\
\hline & DE Crossover Rate & $0.0-1.0$ & Borg, GDE3, MOEA/D, NSGAII \\
\hline & DE Step Size & $0.0-1.0$ & Borg, GDE3, MOEA/D \\
\hline & PCX Parents & $2.0-10.0$ & Borg \\
\hline & PCX Offspring & 1.0-10.0, & Borg \\
\hline & PCX Eta & $0.0-1.0$ & Borg \\
\hline & PCX Zeta & $0.0-1.0$ & Borg \\
\hline & UNDX Parents & $2.0-10.0$ & Borg \\
\hline & UNDX Offspring & $1.0-10.0$ & Borg \\
\hline & UNDX Eta & $0.0-1.0$ & Borg \\
\hline & UNDX Zeta & $0.0-1.0$ & Borg \\
\hline & SPX Parents & $2.0-10.0$ & Borg \\
\hline & SPX Offspring & $1.0-10.0$ & Borg \\
\hline & SPX Epsilon & $0.0-1.0$ & Borg \\
\hline \multirow[t]{3}{*}{ Mutation } & PM Rate & $0.0-1.0$ & $\begin{array}{l}\text { Borg, MOEA/D, } \varepsilon \text {-MOEA, } \varepsilon \text {-NSGAII, } \\
\text { NSGAII }\end{array}$ \\
\hline & PM Distribution Index & $0.0-500.0$ & $\begin{array}{l}\text { Borg, MOEA/D, } \varepsilon \text {-MOEA, } \varepsilon \text {-NSGAII, } \\
\text { NSAII }\end{array}$ \\
\hline & UM Rate & $0.0-1.0$ & Borg \\
\hline \multirow[t]{4}{*}{ Selection } & Neighborhood Size & $0.0-0.2$ & $\mathrm{MOEA} / \mathrm{D}$ \\
\hline & Delta & $0.0-1.0$ & MOEA/D \\
\hline & Eta & $0.0-0.02$ & MOEA/D \\
\hline & Injection Rate & $0.1-1.0$ & Borg, $\varepsilon$-NSGAII \\
\hline PopSize & & $10-1000^{a}$ & AllAlgorithms ${ }^{a}$ \\
\hline$N F E$ & & $10^{4}-10^{5}$ & All Algorithms \\
\hline$L H S$ & & 500 & All Algorithms \\
\hline
\end{tabular}

a The population size range for MOEA/D ranged from 20 to 1000. 


\subsection{Metrics for convergence, consistency, and diversity}

Three metrics were calculated to determine the convergence, consistency, and diversity of each approximation set as in Reed et al. (2013). In single objective optimization, search progress is easily monitored by improvement in the only objective. In multiobjective optimization, this issue becomes more complex, making it important to consider the proximity of an approximation set to the Pareto optimal front or its best known approximation (i.e., convergence to the reference set) as well as its representation of the full extent of tradeoffs (i.e., diversity) (Knowles \& Corne, 2002; Zitzler et al., 2003). Multiple measures are necessary to gain insight to these properties. The three metrics on which this study focuses are generational distance (Van Veldhuizen \& Lamont, 1998), additive epsilon indicator (Zitzler et al., 2003), and hypervolume (Zitzler et al., 2003). We provide a brief summary of each objective below and direct readers interested in more detailed descriptions to Reed et al. (2013); Coello Coello (2007); Zitzler et al. (2003, 2010)

Generational distance (Van Veldhuizen \& Lamont, 1998) is an easy metric to attain as it is purely a measure of convergence and requires only a single solution close to the reference set. Consequently, this metric is meaningful only when an algorithm performs poorly as failure to perform well on generational distance is an abject failure. Generational distance measures the average Euclidean distance of points in an approximation set to their nearest corresponding objective vectors in the reference set. The generational distance is measured between the local Pareto approximate set and the reference (or best known) Pareto approximate set across all objectives.

Additive epsilon indicator (Zitzler et al., 2003) is a harder metric as it is very sensitive to gaps in the approximation set, making it more reflective of the diversity of the approximation set than its convergence. The sensitivity to gaps stems from the fact that the additive epsilon indicator measures the largest distance the approximation set must be shifted to dominate its nearest neighbor in the reference set. If the approximate set has gaps, solutions from other regions must be translated farther distances to dominate the best known approximation, resulting in a dramatically higher additive epsilon indicator value. A good epsilon additive indicator value indicates the approximation set is consistent with the reference set, meaning all portions of the tradeoff are present. Its high penalty for gaps in the approximation set also measures diversity.

Hypervolume (Zitzler et al., 2003) measures the volume of objective space dominated by an approximation set. In this study, the hypervolumes of the approximation sets were normalized to the hypervolume of the reference set. A hypervolume value of one indicates the approximation 
set hypervolume was equivalent to the reference set hypervolume. Hypervolume captures both the convergence and diversity of an approximation set but not consistency. The hypervolume indicator is calculated as the difference in hypervolume between the reference (or best known) Pareto approximate set and the local Pareto approximate set.

The Kruskal-Wallis and Mann-Whitney U nonparametric statistical tests were performed to rank each algorithm's probability of attaining the 75th percentile for each metric. These tests were chosen as they are non-parameteric, meaning they do not make assumptions about the distribution from which the data came. In order to have a sufficiently large population for statistical analysis, the probability of attaining the 75 th percentile was calculated across all parameterizations for each seed of each algorithm. The Kruskal-Wallis test was performed as a six-way filter to determine if there were significant differences in the median probability of attaining the 75th percentile for any algorithm. This test was a useful screen as there would not have been much point in continuing if this test had indicated that the values for all algorithms had been statistically similar. As the KruskalWallis test indicated there was a low probability the medians were equal for all three metrics, a one-tailed pair-wise Mann-Whitney U test was used to determine if the median probability of attaining the 75th percentile for one algorithm exceeded that of another. Algorithms were ranked based on the number of algorithms each outperformed based on the Mann-Whitney U test.

\section{Results and Discussion}

\subsection{Understanding how uncertainty impacts tradeoffs}

The results of this study provide insight into the interdependence between the structure of this four objective problem (i.e., two alternative uncertain natural phosphorus inflow distributions) and its difficulty (i.e., the rigorous MOEA diagnostics). These factors and the Lake Problem's modest computational demands make it a very powerful, yet simple benchmarking problem. Figure 3 shows the global reference sets or best known approximations to the Pareto front for Scenarios 1 (dark blue) and 2 (light blue). The reference sets were obtained from all runs of all of the MOEAs tested. Black arrows designate the directions of increasing preference for each objective. The fourth objective (reliability) is represented by the size of the glyphs where larger spheres represent higher reliability. The ideal point is represented by a large, dark blue star. Although it is not possible to attain the ideal point, it is a reference against which the potential compromises may be evaluated. 

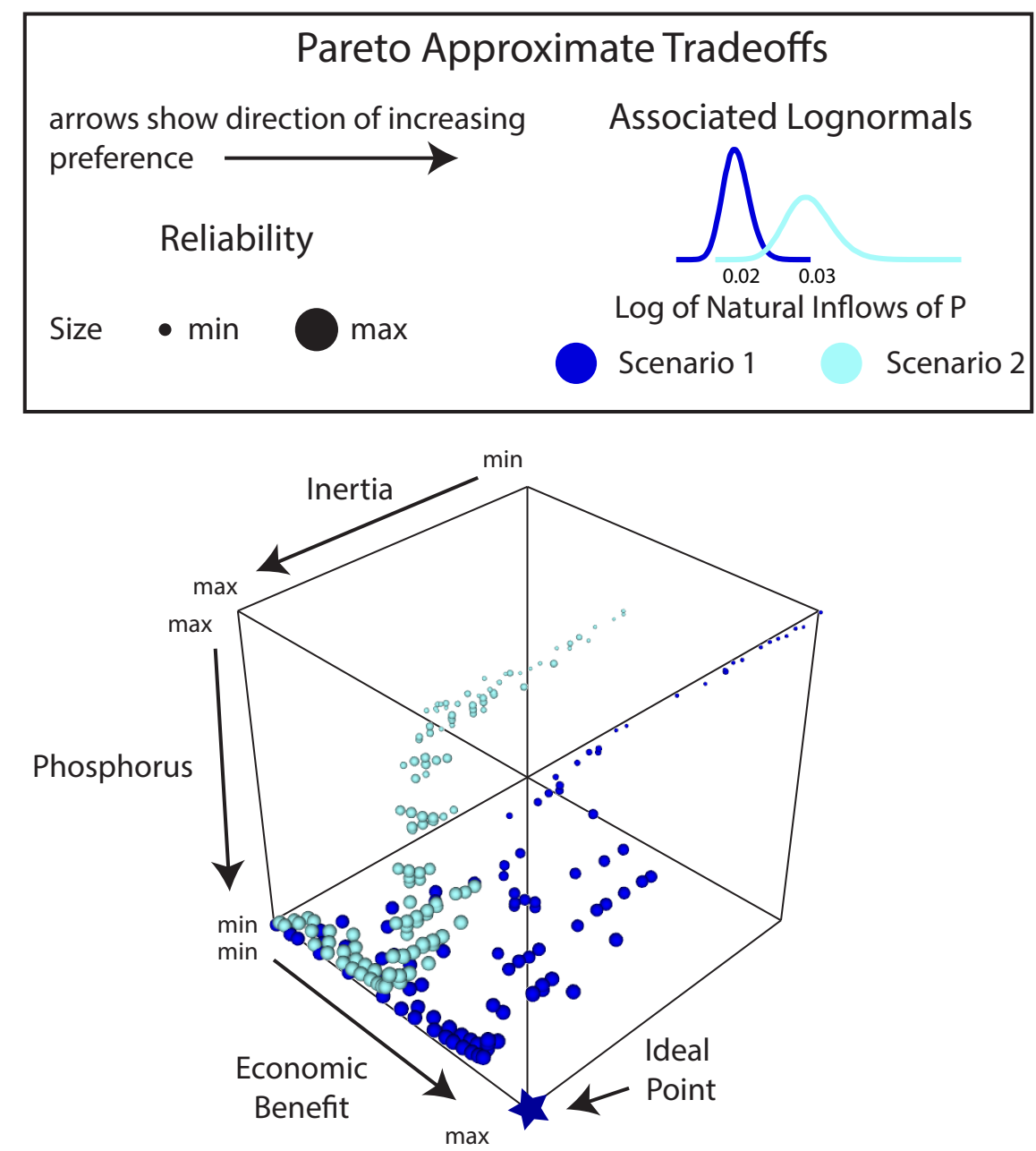

Figure 3: The resulting best known Pareto approximation sets from all runs of all algorithms for Scenario 1 (dark blue) and Scenario 2 (light blue). The star designates the ideal point and arrows along the primary axes show the directions of increasing preference for individual objectives. The size of spheres represents the reliability of management policies in avoiding tipping the lake into a eutrophic state. For both scenarios, decision makers would want large spheres as close to the ideal point as possible.

Scenarios 1 and 2 represent a fairly modest change in the Lake Problem where the sole difference lies in specifying the certainty or knowledge in projecting the natural, uncontrolled phosphorus 
inflows. Scenario 1 has a lower mean phosphorus input and less variability, approximating a case where the decisionmakers are highly knowledgeable in their management actions and can more easily satisfy the $85 \%$ reliability constraint for avoiding phosphorus accumulation above the eutrophic threshold. Scenario 2 has an increased mean and variance for the natural phosphorus leading to a significant reduction in the maximum economic benefits as shown in Figure 3 when comparing the two scenarios' reference sets. Both fronts indicate that solutions resulting in minimal phosphorus in the lake tend to perform better on the reliability and inertia objectives than those that achieved the most economic benefit. Further, while there is a substantial tradeoff between economic benefit and phosphorus in the lake across the majority of the nondominated space, the impact of increased economic benefit on reliability and inertia is quite small except for the highest benefit solutions.

The structural differences in the Scenario 1 and 2 tradeoffs in Figure 3 clearly illustrate the importance of exploring the consequences of the structural uncertainties in how we define environmental management problems (Kasprzyk et al., 2012). Typically, water resources and environmental planning problems focus on a single problem formulation represented by a quantitative model and fixed decision variables; however, better decisions can result from continually updating these formulations and objectives while constructively learning about the problem and stakeholder preferences (Tsoukias, 2008) as doing so shifts the focus of multi-objective optimization from discovering nondominated solutions to developing nondominated problem formulations that improve performance across all objectives as illustrated by Kasprzyk et al. (2012). Consequently, MOEAs must effectively support their users by finding solutions to a variety of problem classes with little sensitivity to their parameters.

\subsection{Effectiveness and Reliability}

As discussed in Sections 4.1-4.2, this study contributes a diagnostic assessment of how well modern MOEAs can discover the tradeoffs for the Lake Problem variants (Figure 3). In this section, we focus on the algorithms' effectiveness and reliability. The black dots shown in panels 4a and $4 \mathrm{~b}$ designate the best overall metrics attained by a single random seed trial of each algorithm across all runs for Scenarios 1 and 2, respectively. The color gradients represent the probability of attaining each threshold-level for each metric (e.g., the probability of capturing $60 \%$ of the best hypervolume). The horizontal axes in both panels of Figure 4 denote the algorithm names as well as their assessed statistical rankings in parentheses. The rankings are based on the Mann-Whitney 
U test for successfully attaining the 75th percentile of each metric. Ideal performance in Figure 4 would be an entirely dark blue bar with a black dot at 100\% designating that an algorithm has a $100 \%$ probability of attaining $100 \%$ of the best possible value. Or put alternatively, a single trial run of an MOEA is expected to be both perfectly reliable and effective. 

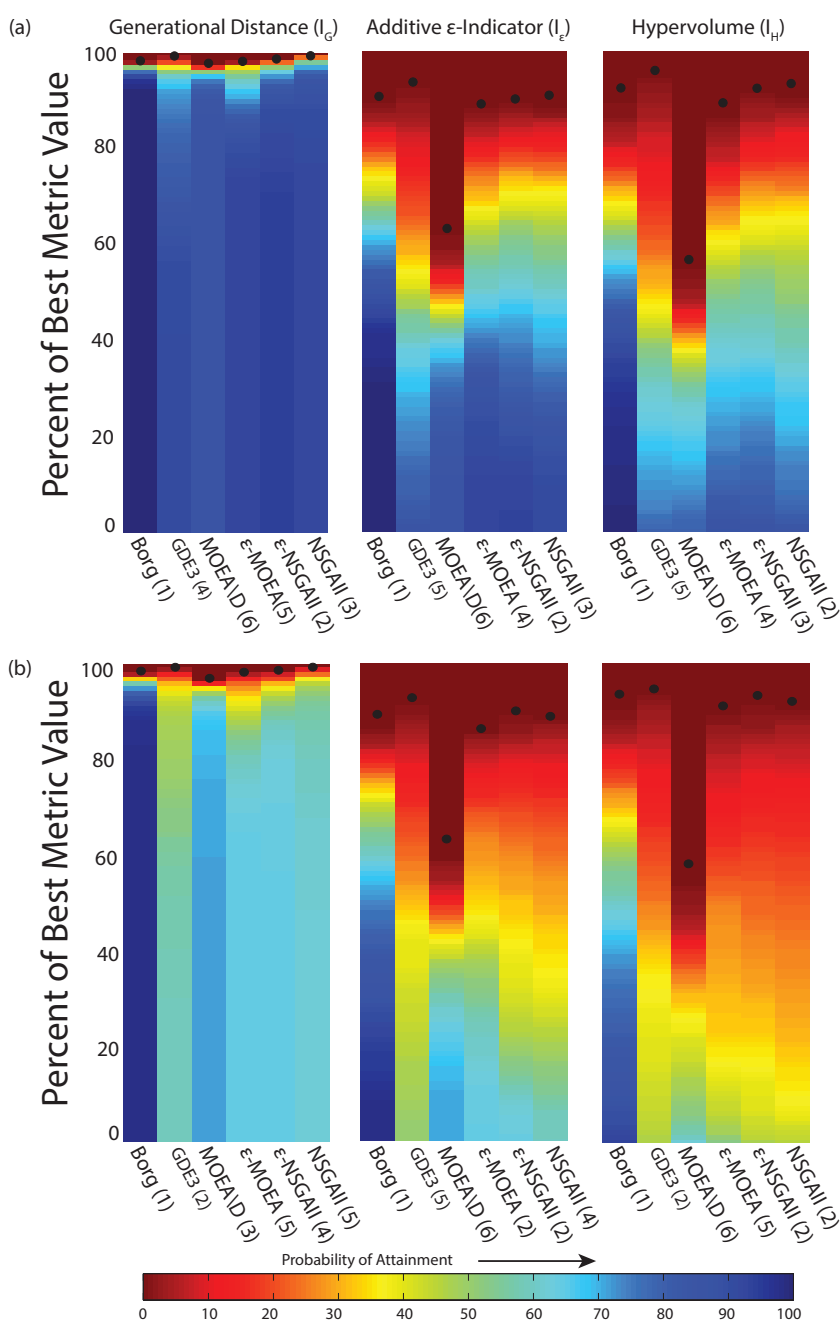

Figure 4: These attainment plots show the best overall performance attained by a single trial of each MOEA (shown as black circles) as well as the probability that a single MOEA trial successfully attains a given percent of the best values for the generational distance, additive $\varepsilon$-indicator, and hypervolume metrics (shown with color shading). Panels (a) and (b) illustrate the attainment performance differences that result from transitioning from Scenario 1 to Scenario 2, respectively. In both panels, the Mann-Whitney U test rankings are shown in parentheses next to each algorithm's name. 
Figure 4 illustrates how reporting the best performance for an MOEA can be misleading without also reporting the probability of attainment. An algorithm that exhibits great performance in its best single run but attains that performance only in a small percentage of its trial runs is of little value to users. Recall from Section 4.2 that each of the metrics reported in Figure 4 measures a different aspect of performance (generational distance-convergence, additive $\varepsilon$-indicator-consistency, and hypervolume-combined convergence and diversity). Overall, generational distance is the easiest of the three metrics, requiring only a single point in an approximation set to be close to the global reference set. This metric is only meaningful for assessing abject MOEA failures (i.e., they could not even get a single point close to the global reference Pareto approximate set). It is interesting to note in comparing the generational distance results in Figure 4a that the modest differences between the algorithms in Scenario 1 change fairly dramatically into severe failures for Scenario 2 in Figure 4b by simply changing to a worse mean phosphorus and higher uncertainty for natural phosphorus inflows. A relatively modest structural change in the problem creates substantial failures in a majority of the MOEAs. Only the Borg MOEA maintained similar attainment values for the generational distance measures.

As expected, transitioning to the more challenging additive $\varepsilon$-indicator and hypervolume metrics exacerbates the MOEAs' failures. Only the Borg MOEA remains relatively reliable with expectation of attaining approximately $60 \%$ of the best values of these metrics in a single trial run. This means that a single seed run of the Borg MOEA across the tested parameterizations would reliably approximate the Lake Problem's tradeoffs even under Scenario 2 (Figure 4b). Alternatively, Scenario 2 causes the remaining algorithms to fail severely. Figure 4 illustrates the best single runs for each MOEA as well as the probabilities of attainment for the average performance by each parameterization, reflecting the results expected by a single random seed trial for each algorithm. Appendix Figure A1 provides attainment maps that reflect each algorithm's performance assuming users would analyze the best resulting approximations across the 50 random seeds used to evaluate each candidate parameterization. MOEA/D consistently demonstrates the weakest performance of the algorithms across all metrics and scenarios. The algorithm exhibits absolute failure in that its best achieved values are only around $60 \%$ of the theoretical maximum for the additive epsilon indicator and hypervolume metrics (Figure $4 \mathrm{a}$ an $4 \mathrm{~b}$ ). This is perhaps surprising considering that it won a world competition in 2008; however, MOEA/D must often be tailored to the problem of interest, is sensitive to the relative scaling of component objectives and has been shown to have 
complex parametric sensitivities that change significantly across different problem classes (Ishibuchi et al., 2009a,b, 2013). MOEA/D's scaling sensitivities are the result of its use of Chebyshev-based aggregations of objectives into a single weighted objective function.

\subsection{Controllability and Efficiency}

The results of Figure 4 explore the effectiveness and reliability of running each of the MOEAs using only a single random seed for both Scenarios 1 and 2 of the Lake Problem. A more standard requirement in the peer reviewed environmental management literature is to run MOEAs for multiple random trials (typically 15-50 random seeds depending on computational demands (Maier et al., 2014; Nicklow et al., 2010; Reed et al., 2013). The results of Figure 4 pose the question of whether or not random seed analysis would significantly improve their performance. To answer this question, Figure 5 presents color control maps for the hypervolume metric for each algorithm for Scenario 1 (Figure 5a) and Scenario 2 (Figure 5b). These control maps present two-dimensional projections of the full suite of the MOEAs' parameters that were globally sampled to assess the impact of the two parameters that strongly influence computational demands: the number of function evaluations (NFE) and the required population size (Reed et al., 2013). The color legend provides a quantitative measure of the percent of the best known reference set's hypervolume captured across 50 random seeds for each of the MOEAs' tested parameterizations. This means that each sampled MOEA parameterization was run for 50 random seed trials and the resulting hypervolume performance as shown in Figure 5 is collected across all of the trials run for each of the MOEAs' sampled parameterization. The results in Figure 5 reflect the standard way MOEAs are used in the literature (i.e., collecting Pareto approximation across multiple random seed trials). Note appendix Figure A2 supplements these results by providing the expected control maps if the MOEAs where run using only a single random trial. 
(a)
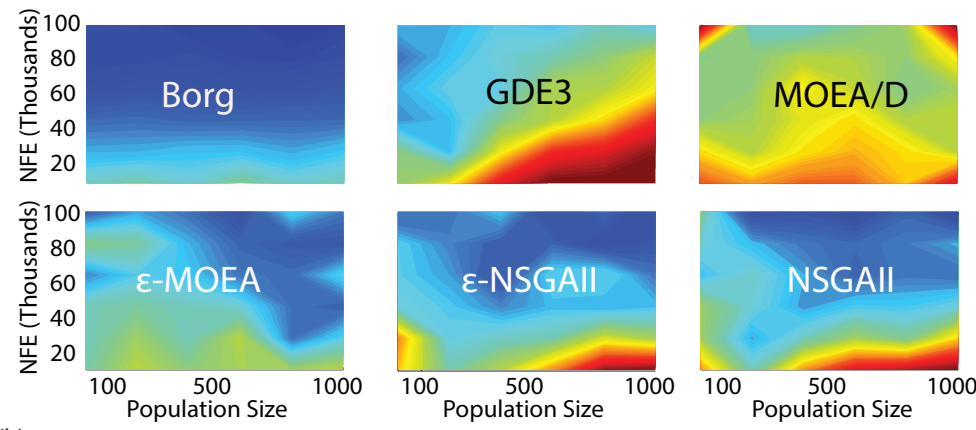

(b)
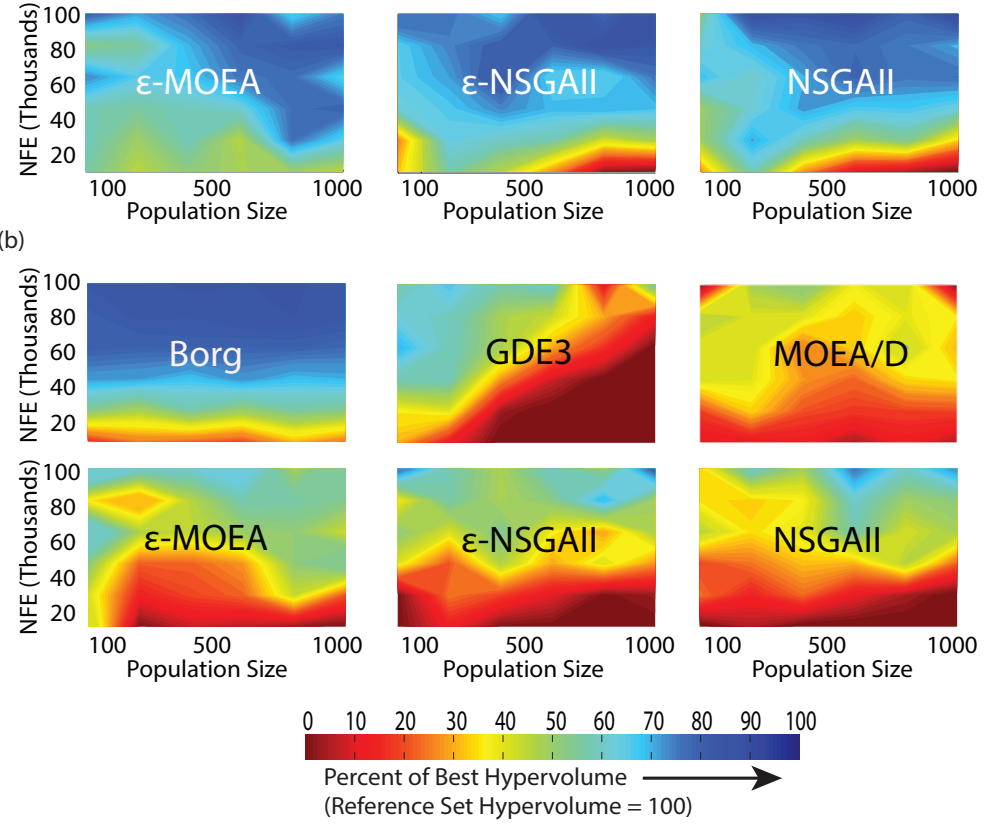

Figure 5: Hypervolume performance control maps capturing controllability and efficiency of each MOEA assuming the use of 50 random seed trials for Scenario 1 in panel (a) and Scenario 2 in panel (b). The color scale represents the percent of the best known global reference set's hypervolume captured by each local 50-seed reference approximation set for each tested parameterization. Although the results are two parameter projections for population size and NFE, their maps are the result of the Latin Hypercube samples of each MOEA's full feasible parameter ranges. Ideal performance is shown in zones of dark blue shading indicating that $100 \%$ of the reference set or best known hypervolume is captured while severely poor performance is designated by dark red. For algorithms with adaptive population sizing, their control maps show their sensitivity to their initial user specified population sizes.

Control maps provide a sense of the "sweet spot" (Goldberg, 2002) for algorithm parameter spaces and, consequently, provide insight into how sensitive each algorithm is to its parameters. As 
noted in Reed et al. (2013) there is a need to shift focus in environmental management applications toward MOEAs that flexibly support the exploration of challenging problem framing hypotheses versus trying to find instances of MOEA parameterizations that work. Often users fail to consider that the number of function evaluations (NFE) is a parameter choice and that it has dramatic effects on computational efficiency as well as the overall decision making process. In Figure 5, an ideal control map would be an entirely dark blue rectangle, indicating that an algorithm attained almost ideal hypervolume regardless of the user-specified parameters and that it is difficult to make an algorithm fail as a result of how it is parameterized. This would also mean that a good approximation to the Pareto front could be attained with the fewest NFE tested (i.e., that the algorithm is highly efficient). The control maps for the Borg MOEA show for Scenario 1 (Figure 5a) and Scenario 2 (Figure 5b) that maximizing algorithm success requires a sufficient investment in NFE. This means that the Borg MOEA's random seed trials successfully converge to the reference set given sufficient time to search regardless of its other parameter values. Overall, the self-adaptive search of the Borg MOEA strongly limits its parametric sensitivities for the Lake Problem as has been seen in several other benchmarked applications (Hadka \& Reed, 2012a; Hadka et al., 2012; Reed et al., 2013). The control maps for Scenario 1 of the Lake Problem in Figure 5a exhibit islands of improved performance that would often be difficult to predict. In Figure 5a, $\varepsilon$-MOEA, $\varepsilon$-NSGAII, and NSGAII demonstrate zones of high performance, yet peak performance would be difficult to attain without some form of a priori global sampling of their parameters. Islands of high performance are highly challenging and designate that the algorithms are not "controllable" (i.e., the "needle-in-the-haystack" parameterization challenge as discussed in Reed et al. (2013)). This means users would struggle to know a priori how to capture the peak performance, limiting the usefulness of these algorithms in decision support. MOEA/D was the worst overall performing algorithm for Scenario 1 of the Lake Problem.

A drastic difference in performance is observable between the two scenarios for all algorithms. The Borg MOEA is still controllable in that a good hypervolume value is attained with an increasing number of function evaluations, but it does demonstrate some failure when too few function evaluations are performed. The increased mathematical difficulty of Scenario 2 is captured in all of the algorithms' control maps and can be observed specifically in the increased NFE required for the Borg MOEA. The other top performing MOEAs in Scenario 1 (Figure 5a) have precipitous reductions in their ability to solve Scenario 2 (Figure $5 \mathrm{~b}$ ). For NSGAII, $\varepsilon$-NSGAII, and $\varepsilon$-MOEA, 
a severe shift from their fairly large swaths of dark blue or large "sweet spots" (Goldberg, 2002) in Scenario 1 to almost none on Scenario 2 is troubling. The difference between the two instances of the Lake Problem is relatively minor and easy to discern relative to what would be expected in more complex real-world environmental management contexts. The results of Figure 5 strongly emphasize the value of the Borg MOEA's self-adaptive search. In terms of self-adaptive search, readers should note that although both the Borg MOEA and $\varepsilon$-NSGAII start with initial user specified population sizes that are adapted, the results in Figure 5a and 5b highlight that only the Borg MOEA is insensitive to its initial population size. The $\varepsilon$-NSGAII has very complex zones of increased performance, and its self-adaptive adjustments of population size are insufficient to overcome search failures for the Lake Problem instances, especially for Scenario 2.

\subsection{Multi-operator cooperative search dynamics}

The results of the diagnostic assessment indicate it is possible for algorithms to fail on even this simple problem, indicating it can be used as an effective benchmarking tool. The shift from Scenario 1 to Scenario 2 in Figures 4 and 5 shows that it becomes harder for algorithms to achieve the best known approximation to the Pareto front if the stochastic phosphorus inflow is assumed to have the higher mean and variability associated with Scenario 2. The adaptive multi-operator search featured by the Borg MOEA makes it less sensitive to its initial parameterization, facilitating its ability to consistently attain high quality approximations to the reference set (Figures 4 and 5). The Borg MOEA is less sensitive to its parameters as it is an evolutionary search framework, consisting of six primary operators, rather than an algorithm. The probability of selecting each operator is updated during the search based on the success of that operator in the previous generation. Figure 6 shows the change in operator probability over time for this problem under both natural phosphorus distributions. These are the operator probabilities averaged across 50 random seeds for the default parameters used by the Borg MOEA. 

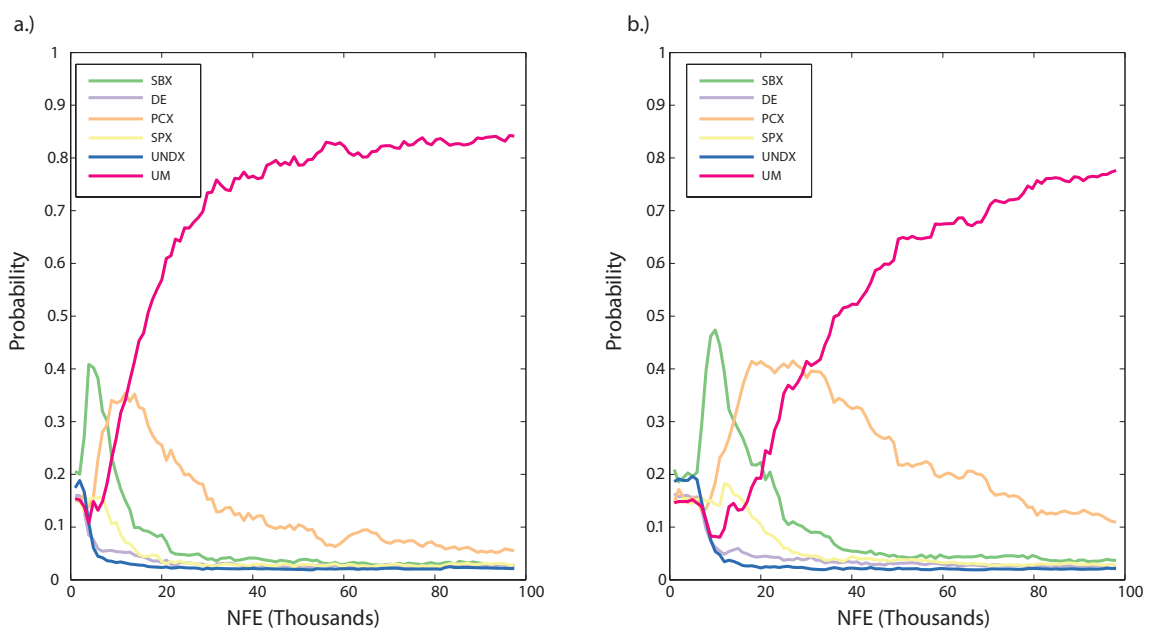

Figure 6: The average Borg MOEA operator dynamics across 50 random seeds for default userspecified parameters on Scenario 1 in panel (a) and Scenario 2 in panel (b) over the first 100 thousand NFE. The Borg MOEA employs six search operators: simulated binary crossover (SBX), differential evolution differencing (DE), parent-centric crossover (PCX), simplex crossover (SPX), unimodal nominal distribution crossover (UNDX), and uniform mutation (UM). High probabilities of using UM in later periods of runs designate when the Borg MOEA has attained a Pareto approximation set that can no longer be refined (i.e., convergence). This phenomenon is illustrated at 20,000 NFE for Scenario 1 in panel (a) and 40,000 NFE in panel (b) for Scenario 2.

Observing the probability associated with each of the operators used by the Borg MOEA over time (Figure 6) provides some useful diagnostic information. For example, in both Figure 6a and 6b, the Borg MOEA is using a cooperative combination of the SBX operator's highly directional (i.e., it assumes decisions are independent) and the PCX operator's rotationally invariant search (i.e., provides perturbations to interdependent decisions in any direction). The Borg MOEA recognizes if it is not discovering new tradeoff solutions and activates its UM operator to ensure that it is not stuck at a false optima. In cases where the algorithm has sufficiently approximated the Pareto front, it cannot make further progress and consequently the UM operator dominates. This gives a clear indication of approximately how many NFE are required to attain a high quality approximation to the Pareto front. This occurs at slightly less than 20,000 NFE for Scenario 1 and 40,000 NFE for Scenario 2. These diagnostics are reflective of the same efficiency trends in the control maps of 
Figure 5. Figure 6 illustrates that this problem did not require much use of the differential evolution (DE) operator, providing insight into the poor performance of the GDE3 algorithm, which serves as a proxy for the DE operator. Simulated Binary Crossover (SBX) was important early in the search for both scenarios (Figure 6) helping to explain why $\varepsilon$-NSGAII, $\varepsilon$-MOEA, and NSGAII, which also use this operator, achieved islands of success on the first scenario (Figure 5a). The more challenging water quality dynamics of Scenario 2 likely increased the degree of inter-dependency between the multi-year control choices when seeking solutions that balance the Lake Problem's economic, water quality, and tipping point objectives. Later in its Scenario 2 operator dynamics, the Borg MOEA shifts to more dominantly use the rotationally invariant PCX and SPX operators. Obviously, these strategies for translating through the decision space for the Lake Problem were not available to the other MOEAs.

\section{Conclusion}

Our study demonstrates that (i) it is challenging to employ multi-objective evolutionary algorithms to find a Pareto approximate front for problems involving uncertain threshold responses and (ii) that it is necessary to consider the choice of algorithm as most fail without carefully tuned parameters. A decision maker does not want to spend valuable time searching for a needle in a haystack to find the right parameterization for an algorithm. Knowing that an algorithm can perform well regardless of its initial parameters allows a decision maker to focus on the greater challenge of formulating the problem appropriately. Our results also indicate that the four objective formulation of the Lake Problem can be a useful benchmarking problem for the use of multi-objective evolutionary algorithms on similar problems as some algorithms fail on even this simple problem, especially when optimization occurs under Scenario 2. Across both scenarios, the Borg MOEA was the only MOEA to demonstrate controllability, reliability, and effectiveness as a result of its adaptive operator selection and adaptive population sizing. The control maps for the Borg MOEA show a marked increase in performance with increases in NFE, meaning that it has excellent controllability, and users do not need to manipulate any of the other parameters to generate a good approximation to the nondominated front for the Lake Problem.

The use of the Lake Problem for benchmarking is also important in that it represents a large class of environmental and water resources problems that have, to date, received relatively sparse attention, namely those characterized by an uncertain threshold. Other aspects of this problem 
that make it a challenging representation of real-world concerns are the large number of decision variables, presence of multiple objectives, and uncertainty. When considering the impact of algorithm choice on the ability to find the Pareto front for similar problems, it is important to consider that these results show algorithms failed with multiple random seeds and up to 100,000 function evaluations. With fewer seeds and NFE, MOEA users face the serious risk of analyzing rather poor approximations to the Pareto front. Choosing an appropriate algorithm can drastically increase the chances that the approximation will be reasonably close to the nondominated front.

Our results also carry implications when one considers the risk-averse nature of most water resources managers. The $15 \%$ risk of failure tolerated in this study is much higher than most water resources decision makers are comfortable accepting (Caldwell \& Characklis, 2014; Characklis et al., 2006; Kasprzyk et al., 2009; Zeff et al., 2014). The fact that the lake eventually flips in both scenarios for this problem is of potential concern and motivates future research on this problem regarding robust decision making (Hadka et al., In-Press), adaptive management through learning and rule-based environmental pollution control strategies (e.g., guide curves) as approaches to decision making in the face of highly uncertain environmental thresholds. Rule-based decision triggers for temporally repeating actions are in fact heuristic simplifications of "optimal control" from a mathematical perspective. The degree that they are sufficient is an area of ongoing research in the control theory literature (Castelletti et al., 2008; Koutsoyiannis \& Economou, 2003; Labadie, 2004). Our present Lake Problem formulation benchmarking results contribute a formal mathematical baseline for future studies to compare the computational and/or decision support consequences of alternative formulations of the Lake Problem.

\section{Acknowledgements}

This work was supported by the National Science Foundation through the Network for Sustainable Climate Risk Management (SCRiM) under NSF cooperative agreement GEO-1240507 as well as the Penn State Center for Climate Risk Managment. Any opinions, findings, and conclusions or recommendations expressed in this material are those of the authors and do not necessarily reflect the views of the US National Science Foundation. 


\section{References}

Admiraal, J. F., Wossink, A., de Groot, W. T., \& de Snoo, G. R. (2013). More than total economic value: How to combine economic valuation of biodiversity with ecological resilience. Ecological Economics, 89, 115-122.

Brill, E. D., Flach, J. M., Hopkins, L. D., \& Ranjithan, S. (1990). MGA: a decision support system for complex, incompletely defined problems. IEEE Trans. Syst., Man, Cybern., 20, 745-757. doi:10.1109/21.105076.

Brock, W. A. (2006). Tipping points, abrupt opinion changes, and punctuated policy change. Punctuated equilibrium and the dynamics of US environmental policy, (pp. 47-77).

Brozović, N., \& Schlenker, W. (2011). Optimal management of an ecosystem with an unknown threshold. Ecological economics, 70, 627-640.

Caldwell, C., \& Characklis, G. W. (2014). Impact of contract structure and risk aversion on interutility water transfer agreements. Journal of Water Resources Planning and Management, 140, 100-111. doi:10.1061/(ASCE)WR. 1943-5452.

Carpenter, S. R., Ludwig, D., \& Brock, W. A. (1999). Management of eutrophication for lakes subject to potentially irreversible change. Ecological applications, 9, 751-771. URL: http://www . esajournals .org/doi/abs/10.1890/1051-0761(1999)009\%5B0751:MOEFLS\%5D2.0.C0\%3B2.

Castelletti, A., Pianosi, F., \& Soncini-Sessa, R. (2008). Water reservoir control under economic, social and environmental constraints. Automatica, 44, 1595-1607. URL: http://www. sciencedirect.com/science/article/pii/S0005109808001271.

Chan Hilton, A. B., \& Culver, T. B. (2005). Groundwater remediation design under uncertainty with genetic algorithms. Journal of Water Resources Planning and Management, 131, 25-34.

Characklis, G., Kirsch, B. R., Ramsey, J., Dillard, K., \& Kelley, C. T. (2006). Developing portfolios of water supply transfers. Water Resources Research, 42. doi:10.1029/2005WR004424.

Clemen, R. T., \& Reilly, T. (2001). Making Hard Decisions with Decision Tools®. Duxbury, Pacific Grove, Calif, USA. 
Coello Coello, C. A. (2007). Evolutionary Algorithms for Solving Multi-Objective Problems. Genetic and Evolutionary Computation (2nd ed.). New York: Springer.

Cohon, J., \& Marks, D. (1975). A review and evaluation of multiobjective programming techniques. Water Resources Research, 11, 208-220.

Deb, K., \& Agrawal, R. B. (1994). Simulated binary crossover for continuous search space. Complex Systems, 9, 1-15.

Deb, K., \& Goyal, M. (1996). A combined genetic adaptive search (geneas) for engineering design. Computer Science and Informatics, 26, 30-45.

Deb, K., Joshi, D., \& Anand, A. (2002a). Real-coded evolutionary algorithms with parent-centric re-combination. In Proceedings of the World Congress on Computational Intelligence (pp. 61-66).

Deb, K., Mohan, M., \& Mishra, S. (2005). Evaluating the epislon-domination based multiobjective evolutionary algorithm for a quick computation of pareto-optimal solutions. Evolutionary Computation Journal, 13, 501-525.

Deb, K., Pratap, A., Agarwal, S., \& Meyarivan, T. (2002b). A fast and elitist multiobjective genetic algorithm: NSGA-II. IEEE Transactions on Evolutionary Computation, 6, 182-197.

Fu, G., \& Kapelan, Z. (2011). Fuzzy probabilistic design of water distribution networks. Water Resources Research, 47. doi:10.1029/2010WR009739.

Giagkiozis, I., \& Fleming, P. J. (2014). Pareto front estimation for decision making. Evolutionary Computation, 22, 651-678.

Goldberg, D. E. (2002). The Design of Innovation: Lessons from and for Competant Genetic Algorithms. Boston: Kluwer Academic Publishers.

Gopalakrishnan, G., Minsker, B., \& Goldberg, D. E. (2001). Optimal sampling in a noisy genetic algorithm for risk-based remediation design. In Bridging the Gap. American Society of Civil Engineers. URL: http://dx.doi.org/10.1061/40569(2001)94. doi:10.1061/40569(2001)94.

Hadka, D., Herman, J., Reed, P., \& Keller, K. (In-Press). Openmordm: An open source framework for many-objective robust decision making. Environmental Modelling and Software, (pp. InPress). 
Hadka, D., \& Reed, P. (2012a). Diagnostic assessment of search controls and failure modes in many-objective evolutionary optimization. Evolutionary Computation, 20, 423-452.

Hadka, D., \& Reed, P. (2012b). MOEAFramework: An open-source Java framework for multiobjective optimization. URL: http://moeaframework.org version 1.17.

Hadka, D., \& Reed, P. (2013). Borg: An auto-adaptive many-objective evolutionary computing framework. Evolutionary computation, 21, 231-259.

Hadka, D., \& Reed, P. (In-Press). Large-scale parallelization of the borg moea for many-objective optimization of complex environmental systems. Environmental Modelling and Software, (pp. In-Press).

Hadka, D., Reed, P. M., \& Simpson, T. W. (2012). Diagnostic assessment of the borg moea for many-objective product family design problems. In Evolutionary Computation (CEC), 2012 IEEE Congress on (pp. 1-10). IEEE.

Haimes, Y. Y., \& Hall, W. A. (1977). Sensitivity, responsivity, stability, and irreversability as multiple objectives in civil systems. Advances in Water Resources, 1, 71-81.

Hall, J., Lempert, R. J., Keller, K., Hackbarth, A., Mijere, C., \& McInerney, D. J. (2012). Robust climate policies under uncertainty: A comparison of robust decision making and info-gap methods. Risk Analysis, . doi:10.111\j.1539-6924.2012.01802.x.

Herman, J. D., Reed, P. M., Zeff, H. B., \& Characklis, G. (In-Press). How should robustness be defined for water systems planning under change? Journal of Water Resources Planning and Management, (p. [In Press]).

Hitch, C. J. (1960). On the Choice of Objectives in Systems Studies. Technical Report P-1955 The RAND Corporation.

Ishibuchi, H., Akedo, N., \& Nojima, Y. (2013). Relation between neighborhood size and moea/d performance on many-objective problems. In Evolutionary Multi-Criterion Optimization (pp. 459-474). Springer.

Ishibuchi, H., Sakane, Y., Tsukamoto, N., \& Nojima, Y. (2009a). Adaptation of scalarizing functions in moea/d: An adaptive scalarizing function-based multiobjective evolutionary algorithm. In Evolutionary Multi-Criterion Optimization (pp. 438-452). Springer. 
Ishibuchi, H., Sakane, Y., Tsukamoto, N., \& Nojima, Y. (2009b). Evolutionary many-objective optimization by nsga-ii and moea/d with large populations. In Systems, Man and Cybernetics, 2009. SMC 2009. IEEE International Conference on (pp. 1758-1763). IEEE.

Kasprzyk, J. R., Reed, P. M., Characklis, G. W., \& Kirsch, B. R. (2012). Many-objective de novo water supply portfolio planning under deep uncertainty. Environmental Modelling and Software, 34, 87-104. doi:10.1016/j.envsoft.2011.04.003.

Kasprzyk, J. R., Reed, P. M., Kirsch, B. R., \& Characklis, G. W. (2009). Managing population and drought risks using many-objective water portfolio planning under uncertainty. Water Resour. Res., 45, n/a-n/a. doi:10.1029/2009wr008121.

Keller, K., Bolker, B. M., \& Bradford, D. F. (2004). Uncertain climate thresholds and optimal economic growth. Journal of Environmental Economics and Management, 48, 723-741.

Keller, K., Yohe, G., \& Schlesinger, M. (2008). Managing the risks of climate thresholds: uncertainties and information needs. Climatic Change, 91, 5-10. URL: http://www.springerlink. com/index/E550754338771K25.pdf.

Kita, H., Ono, I., \& Kobayashi, S. (1999). Multi-parental extension of the unimodal normal distribution crossover for real-coded genetic algorithms. In Congress on Evolutionary Computation (pp. 1581-1588).

Knowles, J., \& Corne, D. (2002). On metrics for comparing nondominated sets. In Evolutionary Computation, 2002. CEC'02. Proceedings of the 2002 Congress on (pp. 711-716). IEEE volume 1.

Köksalan, M. M., Wallenius, J., \& Zionts, S. (2011). Multiple criteria decision making: from early history to the 21st century. World Scientific.

Kollat, J. B., \& Reed, P. M. (2006). Comparing state-of-the-art evolutionary multi-objective algorithms for long-term groundwater monitoring design. Advances in Water Resources, 29, 792-807.

Koutsoyiannis, D., \& Economou, A. (2003). Evaluation of the parameterization-simulationoptimization approach for the control of reservoir systems. Water Resources Research, 39.

Kriegler, E., Hall, J. W., Held, H., Dawson, R., \& Schellnhuber, H. J. (2009). Imprecise probability assessment of tipping points in the climate system. Proceedings of the National Academy of Sciences, 106, 5041-5046. URL: http: 
//www . pnas . org/content/106/13/5041. abstract.

doi:10.1073/pnas. 0809117106. arXiv:http://www.pnas.org/content/106/13/5041.full.pdf+html.

Kukkonen, S., \& Lampinen, J. (2009). Performance assessment of generalized differential evolution 3 with a given set of constrained multi-objective test problems. In Evolutionary Computation, 2009. CEC'09. IEEE Congress on (pp. 1943-1950). IEEE.

Kwadijk, J. C. J., Haasnoot, M., Mulder, J. P. M., Hoogvliet, M. M. C., Jeuken, A. B. M., van der Krogt, R. A. A., van Oostrom, N. G. C., Schelfhout, H. A., van Velzen, E. H., van Waveren, H., \& de Wit, M. J. M. (2010). Using adaptation tipping points to prepare for climate change and sea level rise: a case study in the netherlands. Wiley Interdisciplinary Reviews: Climate Change, 1, 729-740. URL: http://dx.doi.org/10.1002/wcc.64. doi:10.1002/wcc.64.

Labadie, J. W. (2004). Optimal operation of multireservoir systems: State-of-the-art review. Journal of Water Resources Planning and Management, 130, 93-111.

Laumanns, M., Thiele, L., Deb, K., \& Zitzler, E. (2002). Combining convergence and diversity in evolutionary multiobjective optimization. Evolutionary computation, 10, 263-282.

Lempert, R. J., \& Collins, M. (2007). Managing the risk of an uncertain threshold response: Comparison of robust, optimimum, and precautionary approaches. Risk Analysis, 27, 10091026.

Lenton, T. M. (2013). Environmental tipping points. Annual Review of Environment and Resources, $38,1-29$.

Liebman, J. C. (1976). Some simple-minded observations on the role of optimization in public systems decision-making. Interfaces, 6, 102-108.

Maier, H. R., Kapelan, Z., Kasprzyk, J., Kollat, J., Matott, L. S., Cunha, M. C., Dandy, G. C., Gibbs, M. S., Keedwell, E., Marchi, A., Ostfeld, A., Savic, D., Solomatine, D. P., Vrugt, J. A., Zecchin, A. C., Minsker, B. S., Barbour, E. J., Kuczera, G., Pasha, F., Castelletti, A., Giuliani, M., \& Reed, P. M. (2014). Evolutionary algorithms and other metaheuristics in water resources: Current status, research challenges and future directions. Environmental Modelling $\&$ Software, 62, 271-299. URL: http://www.sciencedirect.com/science/article/pii/ S1364815214002679. doi:10.1016/j.envsoft.2014.09.013. 
McInerney, D., Lempert, R., \& Keller, K. (2012). What are robust strategies in the face of uncertain climate threshold responses? Climatic change, 112, 547-568. URL: http://link.springer. com/article/10.1007/s10584-011-0377-1.

Nicklow, J., Reed, P., Savic, D., Dessalegne, T., Harrell, L., Chan-Hilton, A., Karamouz, M., Minsker, B., Ostfeld, A., Singh, A., \& Zechman, E. (2010). State of the art for genetic algorithms and beyond in water resources planning and management. Journal of Water Resources Planning and Management, 136, 412-432.

Pareto, V. (1896). Cours D'Economie Politique. Lausanne: Rouge.

Peterson, G., Carpenter, S., \& Brock, W. A. (2003). Uncertainty and the management of multistate ecosystems: an apparently rational route to collapse. Ecology, 84, 1403-1411. URL: http: //www. esajournals.org/doi/abs/10.1890/0012-9658(2003) 084 [1403:UATMOM] 2.0.C0; 2.

Reed, P., Minsker, B. S., \& Goldberg, D. E. (2003). Simplifying multiobjective optimization: An automated design methodology for the nondominated sorted genetic algorithm-ii. Water Resources Research, 39, n/a-n/a. URL: http://dx.doi.org/10.1029/2002WR001483. doi:10. 1029/2002WR001483.

Reed, P. M., \& Hadka, D. (2014). Evolving many-objective water management to exploit exascale computing. Water Resources Research, (pp. n/a-n/a). URL: http://onlinelibrary.wiley. com/doi/10.1002/2014WR015976/abstract. doi:10.1002/2014WR015976.

Reed, P. M., Hadka, D., Herman, J. D., Kasprzyk, J. R., \& Kollat, J. B. (2013). Evolutionary multiobjective optimization in water resources: The past, present, and future. Advances in Water Resources, 51, 438-456.

Reed, P. M., \& Kasprzyk, J. R. (2009). Water resources management: The myth, the wicked, and the future. Journal of Water Resources Planning and Management, 135, 411-413.

Singh, A., Walker, D. D., Minsker, B. S., \& Valocchi, A. J. (2010). Incorporating subjective and stochastic uncertainty in an interactive multi-objective groundwater calibration framework. Stoch Environ Res Risk Assess, 24, 881-898. doi:10.1007/s00477-010-0384-1. 
Singh, R., Reed, P. M., \& Keller, K. (2015). Many-objective robust decision making for managing an ecosystem with a deeply uncertain threshold response. Ecology and Society, 20. doi:10.5751/ ES-07687-200312.

Smalley, J. B., Minsker, B. S., \& Goldberg, D. E. (2000). Risk-based in situ bioremediation design using a noisy genetic algorithm. Water Resources Research, 36, 3043-3052.

Storn, R., \& Price, K. (1997). Differential evolution - a simple and efficient heuristic for global optimization over continuous spaces. Journal of Global Optimization, 11, 341-359.

Tang, Y., Reed, P., \& Wagener, T. (2006). How effective and efficient are multiobjective evolutionary algorithms at hydrologic model calibration? Hydrology and Earth System Sciences Discussions, 10, 289-307. URL: https://hal.archives-ouvertes.fr/hal-00304845.

Tsoukias, A. (2008). From decision theory to decision aiding methodology. European Journal of Operational Research, 187, 138-161.

Tsutsui, S., Yamamura, M., \& Higuchi, T. (1999). Multi-parent recombination with simplex crossover in real coded genetic algorithms. In Genetic and Evolutionary Computation Conference (GECCO 1999).

Van Veldhuizen, D. A., \& Lamont, G. B. (1998). Evolutionary computation and convergence to a pareto front. In Late breaking papers at the genetic programming 1998 conference (pp. 221-228). Citeseer.

Walker, W. E., Haasnoot, M., \& Kwakkel, J. H. (2013). Adapt or perish: a review of planning approaches for adaptation under deep uncertainty. Sustainability, 5, 955-979.

Wu, X., \& Whittington, D. (2006). Incentive compatibility and conflict resolution in international river basins: A case study of the nile basin. Water Resources Research, 42, W02417.

Zeff, H., Kasprzyk, J., Herman, J., Reed, P., \& Characklis, G. (2014). Navigating financial and supply reliability tradeoffs in regional drought portfolios. Water Resources Research, (p. [In Press]).

Zhang, Q., Liu, W., \& Li, H. (2009). The performance of a new version of moea/d on cec09 unconstrained mop test instances. In IEEE Congress on Evolutionary Computation (pp. 203208). 
Zhang, Q., \& Suganthan, P. N. (2009). Final report on cec09 moea competition. In Congress on Evolutionary Computation (CEC 2009).

Zitzler, E., Thiele, L., \& Bader, J. (2010). On set-based multiobjective optimization. Trans. Evol. Comp, 14, 58-79. URL: http://dx.doi .org/10.1109/TEVC. 2009.2016569. doi:10.1109/TEVC. 2009.2016569.

Zitzler, E., Thiele, L., Laumanns, M., Fonseca, C., \& da Fonseca, V. (2003). Performance assessment of multiobjective optimizers: an analysis and review. Evolutionary Computation, IEEE Transactions on, 7, 117-132. doi:10.1109/TEVC.2003.810758. 
(a)

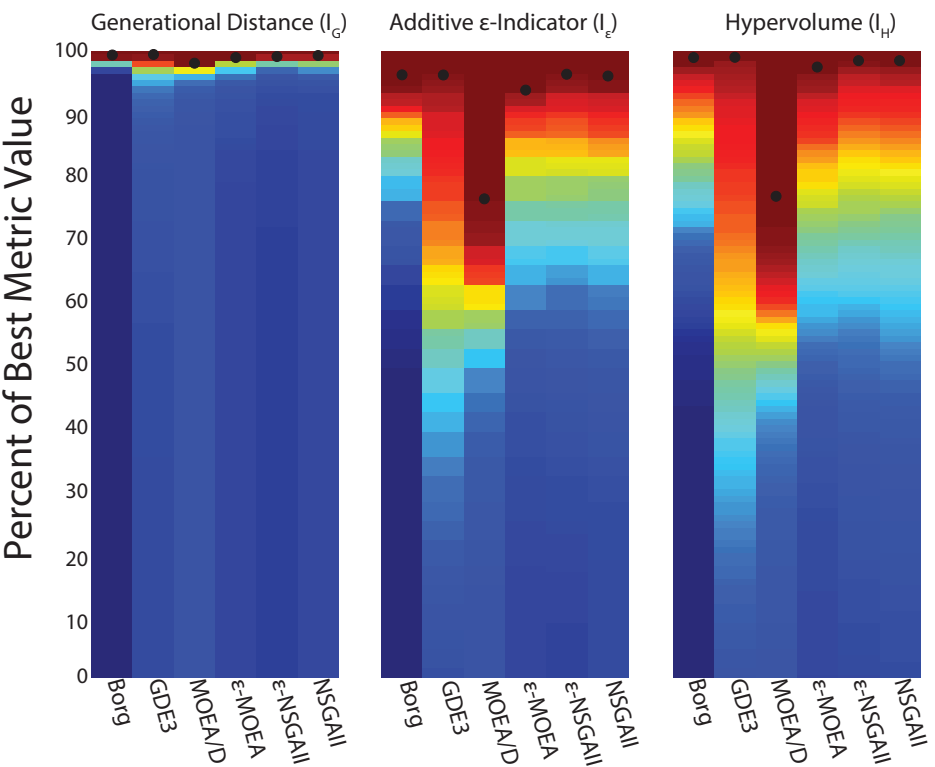

(b)

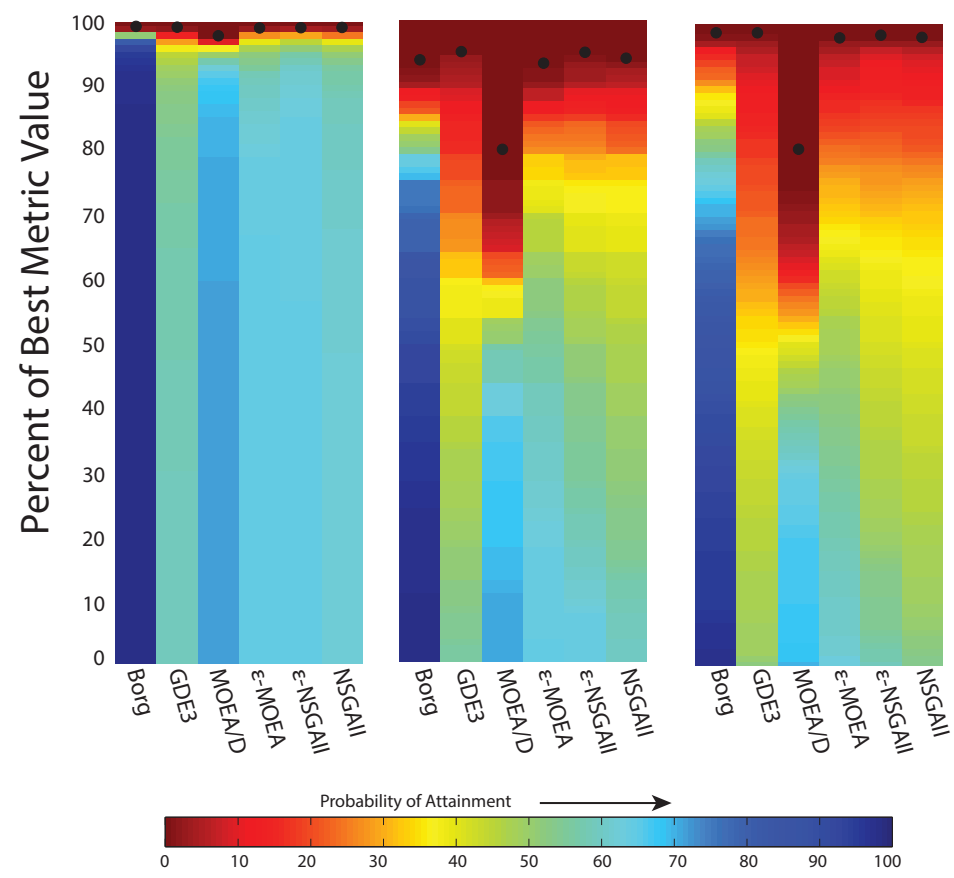

Figure A1: These attainment plots show the best overall performance attained by the use of 50 random seed trials for each MOEA (shown as black circles) as well as the probability that random seed analysis of a single MOEA parameterization successfully attains a given percent of the best values for the generational distance, additive $\varepsilon$-indicator, and hypervolume metrics (shown with color shading). Panels (a) and (b) illustrate the attainment performance differences that result from transitioning from Scenario 1 to Scenario 2, respectively. 
(a)
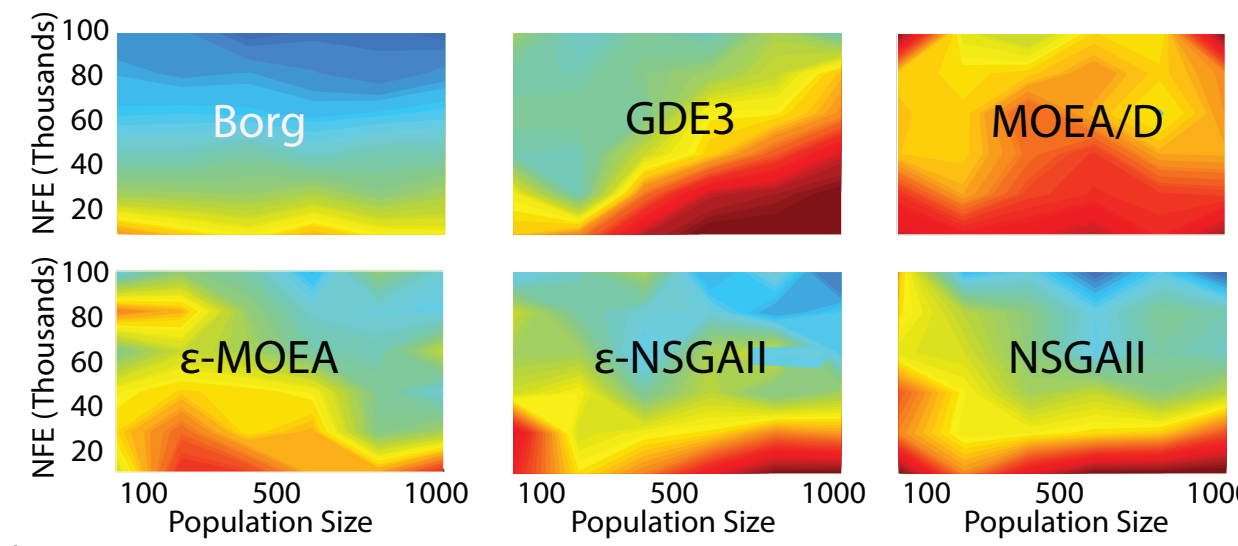

(b)
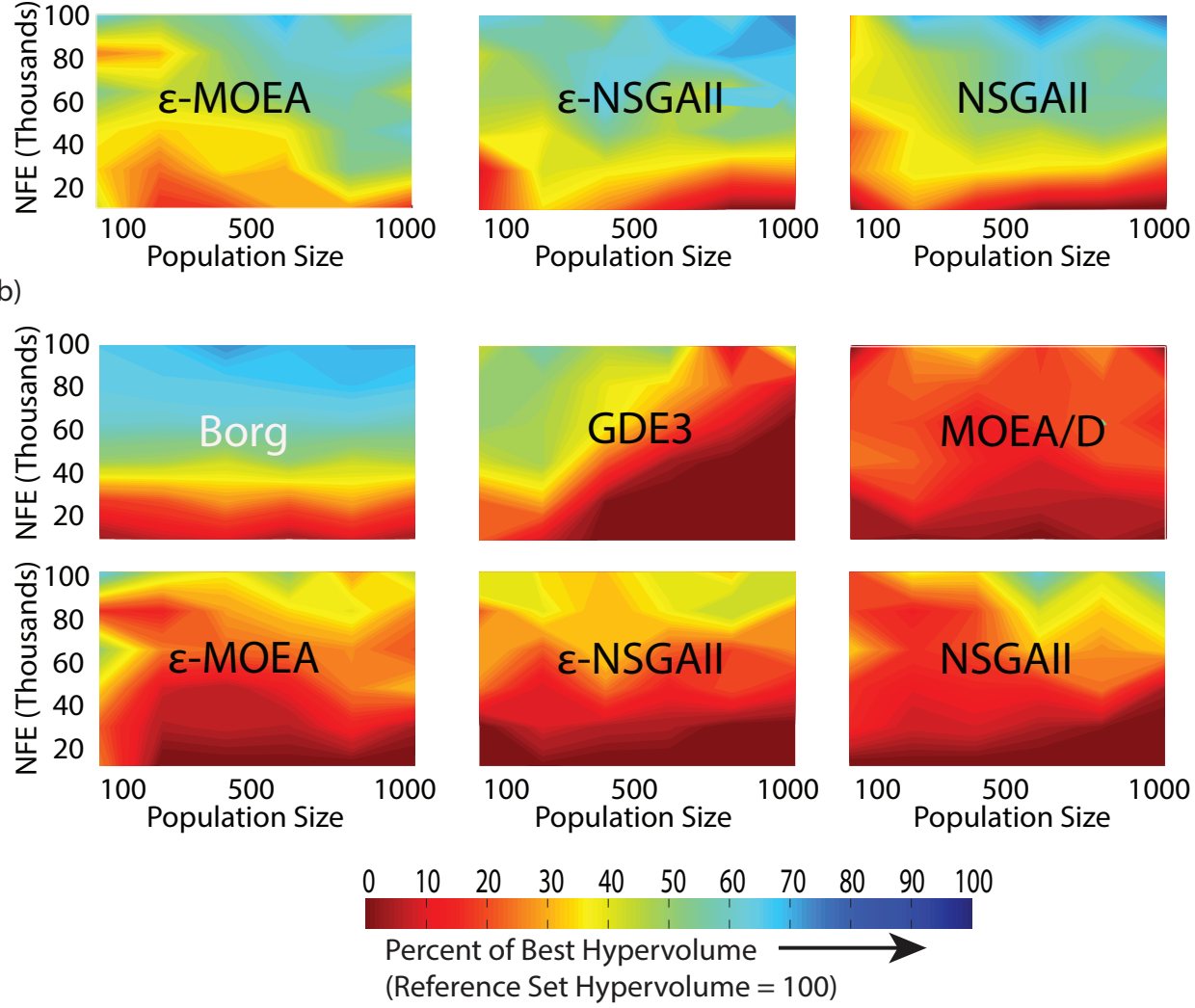

Figure A2: Hypervolume performance control maps capturing controllability and efficiency of each MOEA assuming a single random seed trial for Scenario 1 in panel (a) and Scenario 2 in panel (b). The color scale represents the percent of the best known global reference set's hypervolume captured by each expected single seed approximation set for each tested parameterization. Although the results are two parameter projections for population size and NFE, their maps are the result of the Latin Hypercube samples of each MOEA's full feasible parameter ranges. Ideal performance is shown in zones of dark blue shading indicating that $100 \%$ of the reference set or best known hypervolume is captured while severely poor performance is designated by dark red. For algorithms with adaptive population sizing, their control maps show their sensitivity to their initial user specified population sizes. 